\title{
Stuttgarter Burgfrieden und Burgfriedensbezirk im Spiegel der württembergischen Hofordnungen
}

\author{
Von Anja Kircher-Kannemann
}

\section{Einleitung}

Burgfrieden und Burgfriedensbezirke haben in der historischen Forschung bislang selten viel Beachtung gefunden. Dem Lexikon des Mittelalters ist der Burgfrieden nicht einmal einen eigenen Eintrag wert ${ }^{1}$, und auch das große zweibändige Werk „Die Burgen im deutschen Sprachraum“ ${ }^{2}$ widmete dem Burgfrieden keinen eigenen Artikel ${ }^{3}$.

Dabei waren Burgfriedensbezirke vom Mittelalter bis hinein in die Neuzeit eigenständige Rechtsbezirke mit zum Teil sehr stark von anderen Rechtsbereichen abweichenden Regelungen ${ }^{4}$ und sie waren vor allem nicht nur Rechtsbereiche für eine verschwindend kleine Minderheit, sondern galten teilweise für hunderte oder

${ }^{1}$ Lexikon des Mittelalters, 9 Bde., München/Zürich, 1980-1998 (künftig: LexMA). Lediglich im Artikel „Burg“ findet sich der Burgfrieden kurz erwähnt und im Artikel „Ganerben" wird auf eben diesen verwiesen, s. H.-P. Baum, Art. Burg, C 1 [3], in: LexMA 2, Sp. 968 -971, hier Sp. 970 f. und K.-F. Krieger, Art. Ganerben, Ganerbschaft, in: LexMA 4, Sp. 1105.

${ }^{2}$ Die Burgen im deutschen Sprachraum. Ihre rechts- und verfassungsgeschichtliche Bedeutung, hg. von Hans Patze, 2 Bde., Sigmaringen 1976. Nur Hans-Martin Maurer ging in seinem Artikel über die Rechtsverhältnisse der hochmittelalterlichen Adelsburgen auf den Burgfrieden näher ein, s. Hans-Martin MAURER, Rechtsverhältnisse der hochmittelalterlichen Adelsburgen vornehmlich in Südwestdeutschland, in: Die Burgen im deutschen Sprachraum. Ihre rechts- und verfassungsgeschichtliche Bedeutung, hg. von Hans Patze, Sigmaringen 1976, Bd.2, S.77-190, hier S. 104-116.

3 Allerdings finden sich in diesem Werk einige Artikel, die sich mit der Thematik des Burgfriedens auseinandersetzen, neben dem Artikel von Maurer auch Hans Patze, Rechts- und verfassungsgeschichtliche Bedeutung der Burgen in Niedersachsen, ebd., Bd.1, S.515-564, hier S.543-552, und Francois Rapp, Zur Geschichte der Burgen im Elsaß mit besonderer Berücksichtigung der Ganerbenschaften und der Burgfrieden, ebd., Bd.2, S. 229-248, hier S. 243-248.

${ }^{4}$ Zumeist waren die Strafen in den Burgfriedensbezirken deutlich schärfer, s. Kurt Treusch von Buttlar, Das tägliche Leben an den deutschen Fürstenhöfen des 16. Jahrhunderts, in: Zeitschrift für Kulturgeschichte (1897) S.1-41, hier S.14; Friedrich Karl von Moser, Teutsches Hof-Recht in zwölf Büchern, 2 Bde., Frankfurt/Leipzig 1761, Bd.2, 
sogar tausende von Personen, die am Hof eines Fürsten tätig waren oder diesen besuchten.

Es ist in der Tat so, dass selbst die Quellen - jenseits der eigentlichen schriftlich fixierten Burgfriedensordnungen - vielfach über die Burgfriedensbezirke schweigen. Weder über das in ihnen geltende Recht erfährt man in aller Regel viel, noch und vor allem über ihre Ausdehnung. Zumeist wird nur darauf verwiesen, dass es einen solchen Burgfrieden mit entsprechendem Bezirk gibt ${ }^{5}$. Selbst die berühmteste und opulenteste frühneuzeitliche Enzyklopädie, der „Zedler“, schweigt zum Thema. Zwar gibt es einen Eintrag „Burgfrieden“, dieser verweist auf einen Artikel über „Ganerben“, der jedoch ins Leere läuft ${ }^{6}$, wie dies im „Zedler“ leider häufiger geschieht.

Gänzlich anders sieht die Situation für den Stuttgarter Hof aus. Hier befinden wir uns in einer ausnehmend glücklichen Situation, was die Quellenlage betrifft, denn die württembergischen Hofordnungen gehen über Jahrhunderte hinweg detailliert auf den herrschenden Burgfrieden ein und vor allem auch (und dies ist besonders ungewöhnlich und einzigartig), geben sie die genauen Grenzen des in Stuttgart geltenden Burgfriedensbezirkes an ${ }^{7}$.

S. 800; Andreas Elias Rossmann, Vom Hofrechte, in: Erlangische gelehrte Anzeigen (1749) S. 225-232, hier S.228, und Patze (wie Anm.3) S. 546.

5 S. Georg Engelhart Löhneysen, Aulico Politica. Darin gehandelt wird I. Von Erziehung und Information Junger Herrn, II. Vom Ampt/Tugend und Qualiter der Fürsten/und Bestellung derselben Räthe und Officirer. III. Von Bestellung der Concilien, die ein Fürst in seinem Lande haben muß, Remlingen 1622, S. 345.

${ }^{6}$ S. Grosses vollständiges Universal-Lexicon Aller Wissenschafften und Künste, 64 Bde., Halle/Leipzig 1732-1750, hier Bd.4, S. 1968.

7 Folgende Hofordnungen wurden berücksichtigt: Hofordnung Graf Ulrich V., 1478, in: Heidrun Hofacker, Kanzlei und Regiment in Württemberg im späten Mittelalter, Diss. Phil., Filderstadt 1989, S. 224 ff.; Hofordnungen Herzog Christoph, 1549 und 1550, in: Arthur Kern, Deutsche Hofordnungen des 16. und 17. Jahrhunderts (Denkmäler der deutschen Kulturgeschichte, 2. Abt., Bd.1-2), 2 Bde., Berlin 1905-1907, hier Bd.2, S.141 f., und Bd.2, S.142f.; Hofordnungen Herzog Johann Friedrich, 1611, 1614 und 1618, ebd.; Hofordnung Herzog Eberhard III., 1660, ebd.; Hofordnung Herzog Eberhard Ludwig, 1685, 1696 und 1711, ebd. und Moser (wie Anm. 4) Bd.1, S. 54-73; Hofordnung Herzog Ludwig Eugen von Württemberg-Teck, Stuttgart 1794, Dezember 8, WLB Stuttgart, Wirt. R. fol.129-138; Hofordnung Herzog Friedrich II. von Württemberg-Teck, Stuttgart 1798, April 20, WLB Stuttgart Wirt. R. fol.130-8, verfügbar unter http://digital.wlb-stuttgart.de/ purl/bsz394219473 (Zugriff 8. 8. 2015), und Hofordnung König Friedrich von Württemberg 1807, in: A. L. Reyscher (Hg.), Vollständige, historisch und kritisch bearbeitete Sammlung der württembergischen Gesetze, Tübingen 1839, S.87-93. Die jüngste und letzte Hofordnung für Württemberg stammt aus dem Jahr 1818 von König Wilhelm I., in: Das Regierungs-Blatt für das Königreich Württemberg im Auszuge. Eine Sammlung sämmtlicher in den Regierungs-Blättern des Königreichs Württemberg vom Jahre 1806 an enthaltenen, noch ganz oder theilweise gültigen Gesetze, Verordnungen etc., mit erläuternden Anmerkungen und einem Haupt-Register 1840, S.71-77. 
Ähnlich wie mit der Thematik der Burgfrieden und Burgfriedensbezirke sah es in der Forschung auch lange Zeit in Hinblick auf die Quellengattung der Hofordnungen aus: Obwohl sie zahlreiche wichtige Informationen über das Leben an Höfen, über deren Struktur und Aufbau beinhalten, wurden sie über Jahrzehnte hinweg von der Forschung vernachlässigt; ein Umstand, der sich erst durch die Arbeit der Residenzen-Kommission der Akademie der Wissenschaften in Göttingen und ihres Leiters - Werner Paravicini ${ }^{8}$ - langsam veränderte ${ }^{9}$. Gerade deshalb erscheint die Untersuchung der württembergischen Hofordnungen so wichtig, denn in ihnen vereinen sich beide Quellengattungen: Burgfriedensordnungen und Hofordnungen.

\section{Burgfrieden und Burgfriedensbezirk}

Burgfrieden und Burgfriedensbezirke erscheinen in der heutigen historischen Forschung fast ausschließlich als mittelalterliche Phänomene. Werden sie überhaupt thematisiert, dann in Verbindung mit Ganerbenburgen, jenen Burgen also, die im Besitz mehrerer Familien oder Eigentümer waren und bei denen im besonderen Maße das Risiko von Auseinandersetzungen und Fehden bestand, durch die die Burg und ihr Personal Schaden nehmen konnten ${ }^{10}$.

Die speziellen Friedensverträge, die für eben diese Burgen geschlossen wurden, wurden als Burgfriedensverträge oder schlicht als Burgfrieden bezeichnet. Ins-

8 Werner Paravicini veröffentlichte gemeinsam mit Holger Kruse die burgundischen Hofordnungen, s. Holger Kruse/Werner Paravicini, Die Hofordnungen der Herzöge von Burgund, Bd.1: Herzog Philipp der Gute 1407-1467 (Instrumenta, Bd.15), Ostfildern 2005, und Holger Kruse, Die Hofordnungen Herzog Philipps des Guten von Burgund, in: Höfe und Hofordnungen 1200-1600. 5. Symposium der Residenzen-Kommission der Akademie der Wissenschaften in Göttingen, Sigmaringen 5. bis 8. Oktober 1996 (Symposium der Residenzen-Kommission der Akademie der Wissenschaften in Göttingen, Bd.5), hg. von Holger Kruse/Werner Paravicini, Sigmaringen 1999, S.141-165.

9 Den Anfang machte ein Symposium im Jahr 1996 mit dem Titel „Höfe und Hofordnungen“, s. Höfe und Hofordnungen (wie Anm.8). Das ursprünglich gesteckte Ziel, die deutschen Hofordnungen in ihrer Gesamtheit zu erfassen, konnte jedoch nicht erreicht werden. Im Zusammenhang mit der Arbeit der Residenzen-Kommission entstanden jedoch zwei große Hofordnungseditionen, s. Klaus Flink/Bert Thissen, Die klevischen Hofordnungen (Rechtsgeschichtliche Schriften, Bd. 9), Köln 1997, und Margarete BruckHaus/Brigitte Kasten, Die jülich-kleve-bergischen Hof-, Hofämter- und Regimentsordnungen 1456/1521 bis 1609 (Residenzenforschung, Bd. 26), Ostfildern 2015. Außerdem erschien im Jahr 2011 eine Edition der kaiserlichen Hofordnungen, s. Jakob WüHRER/Martin SCHEUTZ, $\mathrm{Zu}$ Diensten Ihrer Majestät. Hofordnungen und Instruktionsbücher am frühneuzeitlichen Wiener Hof (Quelleneditionen des Instituts für Österreichische Geschichtsforschung, Bd.6), Wien 2011.

10 S. Werner Ogris, Art. Ganerben, in: Handwörterbuch zur deutschen Rechtsgeschichte, Bd.1, Berlin ${ }^{2} 2008$, S. $1928-1930$. 
besondere in Lexika, wie dem „Lexikon des Mittelalters“ oder auch dem „Handwörterbuch für deutsche Rechtsgeschichte“, sowie in Werken der Verfassungsgeschichte, findet sich der Begriff Burgfrieden in aller Regel einzig in diesem Zusammenhang ${ }^{11}$. Gelegentlich wird der Begriff auch in Verbindung mit Städten und Klöstern genannt und bezeichnet hier deren eigenen „Friedensbereich“12. So verwundert es nicht, dass die einzigen Einzeluntersuchungen, die zum Thema vorliegen, eben genau diesen speziellen Fall des Burgfriedensvertrages auf Ganerbenburgen genauer untersuchen ${ }^{13}$.

Nur selten wird darauf hingewiesen, dass der Begriff Burgfrieden aber noch weitere Bedeutungen haben kann, die ausgesprochen verschiedener Natur sind. Einzig das „Deutsche Rechtswörterbuch“ führt alle Bedeutungen auf, die sich hinter dem Begriff Burgfrieden verbergen ${ }^{14}$ : So wird der Begriff zum einen zeitweilig als Synonym für „Bergfried“ verwendet und bezeichnet so schlichtweg ein Gebäude ${ }^{15}$; zum anderen meint er allgemein den Frieden, der auf einer Burg eingehalten werden muss. Dies betrifft vor allem den Frieden auf sog. Ganerbenburgen, die mehreren Eigentümern gehörten. Zusätzlich ist aber eben auch der „erhöhte Frieden“ an fürstlichen Höfen gemeint, um den es eben hier konkret gehen soll und der in den Hofordnungen, vor allem den württembergischen, thematisiert wird. Die vierte Bedeutung des Begriffs „Burgfrieden“ meint den befriedeten Bezirk um eine Burg oder aber auch um eine Stadt, der zumeist auch ein eigener Immunitäts- bzw. Gerichtsbezirk war und in diesen Ausführungen als „Burgfriedensbezirk" bezeichnet wird"

Meist werden diese unterschiedlichen Bedeutungen des Begriffs nicht klar voneinander geschieden, und so kommt es zeitweilig zu verwirrenden Definitionsversuchen, die zum einen den Burgfrieden als „eine Rechtsform zur Regelung des Zusammenlebens auf einer Burg“ bezeichnen und dann als „Synonym für einen Teilungsvertrag und das Bemühen um Frieden“, ohne aber darauf hinzuweisen, dass der „Burgfrieden“ mehrere Bedeutungen haben konnte, und diese nicht in genannter Form ineinander verschwimmen ${ }^{17}$.

11 S. Eugen Haberkern/Joseph Friedrich Wallach (Hg.), Hilfswörterbuch für Historiker. Mittelalter und Neuzeit, 2 Bde., Tübingen ${ }^{71987}$; BAUm (wie Anm.1) und Reinhard Sснмiтt, Art. Burgfriede, in: Handwörterbuch zur deutschen Rechtsgeschichte, Bd.1, Berlin ${ }^{2} 2008$, S. 765-766, sowie Krieger (wie Anm. 1) und Hans K. Schulze, Grundstrukturen der Verfassung im Mittelalter, 3 Bde., Stuttgart 1985-1998, hier Bd.2, S. 116.

12 Ebd., Bd.2, S. 115.

13 S. etwa Margret SäNGer, Die Burgfrieden der Grafen von Katzenelnbogen, in: Blätter für deutsche Landesgeschichte (1980) S. 189-234.

14 Art. Burgfrieden, in: Deutsches Rechtswörterbuch, Weimar 1912, Bd. 2, S. 617-619.

15 Vgl. Rapp (wie Anm.3) S. 243.

$16 \mathrm{Zu}$ diesen unterschiedlichen Definitionen vgl. auch Haberkern/Wallach (wie Anm.11), Bd.1, S. 90.

17 Schmitt (wie Anm.11). 
Außer Acht gelassen wird dabei überdies, dass sich die Bedeutung des Wortes „Burgfrieden“ von seiner Entstehung im Mittelalter bis hin zur Neuzeit, die hier behandelt wird, stark veränderte ${ }^{18}$. Wie kommt es, dass dieses eine Wort so viele und vor allem auch so unterschiedliche Bedeutungen haben kann?

Dieser Frage ging vor einigen Jahren bereits Hans-Martin Maurer nach, und auch er stützte sich dabei primär auf württembergische Quellen. Ausgehend von zwei Untersuchungen, die zum Ende des 19. bzw. zu Beginn des 20. Jahrhunderts entstanden waren, versuchte Maurer die Entstehung und die Veränderung des Begriffs Burgfrieden zu ergründen. Die Thesen, die Keutgen und Hirsch aufgestellt hatten ${ }^{19}$, besagten, dass Burgen im 10. und 11. Jahrhundert eine Form des „erhöhten Friedens besessen hätten [...] kraft dessen alle Burgen königlich gewesen seien. “20 Maurer stellte die Frage, ob es jenen „erhöhten Frieden“ überhaupt je gegeben habe und ob die Erscheinungen des Burgfriedens in der Frühen Neuzeit eventuell auf diesen zurückbezogen werden könnten.

Betrachtet man die Rechtsquellen des Mittelalters, abseits der reinen Burgfriedensordnungen, dann stellt man schnell fest, dass der Begriff „Burgfrieden“ quasi nie erscheint. Die Rechtsspiegel des 13. Jahrhunderts, die Maurer untersuchte, verwendeten weder den Begriff, noch konstatierten sie in irgendeiner Form einen erhöhten Frieden für Burgen. Lediglich andere urkundliche Quellen verwendeten den Begriff, bezogen ihn aber ausnahmslos auf Städte und Klöster ${ }^{21}$. Dies erklärt sich daraus, dass die Burg schlicht als „Haus“ angesehen wurde und daher lediglich den gleichen Frieden genoss wie jedes andere Haus auch. „Der Unterschied zwischen Haus und Burg liegt demnach nur in einer räumlichen Ausweitung des Friedens, denn die Burg ist ein Gebäudekomplex mit Höfen und Nebenbauten innerhalb einer Umwehrung. “22

Erst gegen Ende des 13. Jahrhunderts kam der Begriff Burgfrieden auch für Burgen auf und bezeichnete fortan einen freiwillig geschlossenen Vertrag zwischen mehreren Parteien bezüglich einer besitzrechtlich geteilten Burg. Diese Bedeutung setzte sich durch, und so wurde der Begriff im Spätmittelalter, spätestens seit dem Beginn des 14. Jahrhunderts, nahezu ausschließlich benutzt ${ }^{23}$.

Auf einem Frieden „besonderer Qualität“ beruhten die Burgfriedensverträge jedoch nicht, sondern es handelt sich vielmehr um „genossenschaftlich vereinbarte

18 Maurer (wie Anm. 2) S. 105.

19 S. hierzu F. Keutgen, Untersuchungen über den Ursprung der deutschen Stadtverfassung. Habilitationsschrift, Leipzig 1894, und H. Нirsch, Die Klosterimmunität seit dem Investiturstreit. Untersuchungen zur Verfassungsgeschichte des deutschen Reiches und der deutschen Kirche, Weimar 1913, ND Köln/Graz 1967.

${ }^{20}$ Maurer (wie Anm. 2) S. 104.

21 Ebd., S.108; vgl. Schulze (wie Anm.11), Bd.2, S.115.

22 Maurer (wie Anm. 2) S. 106.

23 Ebd., S. 109 und S.111 f.; Schulze (wie Anm.11), Bd.2, S.116, vgl. auch SäNger (wie Anm.13). 
Friedensverträge [...] [die] den Frieden zwischen den Eigentümern erhalten [sollten] und zwar auch, wenn diese in Fehden verwickelt waren [...]. “24

Wie bereits angedeutet, bezog sich der Burgfrieden nicht nur auf ein einzelnes Gebäude, sondern umfasste in aller Regel einen Gebäudekomplex. Doch wie groß war dieser Bereich, in dem der Burgfrieden galt? Wie sahen die Rechtsverhältnisse im Burgfriedensbezirk tatsächlich aus, und wer hatte dort die Strafbefugnisse? Auf diese Fragen bietet die Sekundärliteratur nur selten eine Antwort. Die Ausdehnung eines solchen Burgfriedensbezirkes wird in aller Regel nicht näher erläutert, und es heißt eher lapidar, dass er den die „Burg umgebenden Bereich“ 25 bezeichnete.

Erst ein Blick in die Quellen gibt über die Ausdehnung des Burgfriedensbezirkes näheren Aufschluss und zeigt, dass diese Bereiche durchaus ein deutlich größeres Gebiet umfassen konnten, als nur die Burg mit ihren Umwehrungen ${ }^{26}$ : François Rapp wies in seiner Untersuchung über Burgen im Elsass für den Friedensbezirk von Nideck im Jahre 1422 nach, dass dieser etwa 40 Quadratkilometer umfasste ${ }^{27}$. Dies wiederspricht der landläufigen Annahme, dass Burgfriedensbezirke einem „in einem engen Abstand um die Burg verlaufenden Kreis“ entsprachen ${ }^{28}$. Sie konnten sehr wohl also auch deutlich größer sein und sich auf „vor der Burg gelegene Siedlung[en] “ erweitern ${ }^{29}$.

Einen ziemlich guten Eindruck von der Größe eines Burgfriedensbezirkes liefert Friedrich Karl von Moser in seinem „Teutschen Hof-Recht“: Der Burg-Fride begreifft in sich das Wobn-oder Residenz-Schloß und alle in dessen Umfang begriffene Gebäude, daber auch die anstossenden Gärten, Oeconomie, und Lust-Gebäude dazu gehören ${ }^{30}$. Über den Umfang hinaus beschrieb Moser auch die Art der Kennzeichnung des Burgfriedensbezirkes: Die Wabrzeichen und Merckmale, wie weit die Gränzen des Burg-Fridens sich erstrecken, seynd entweder durch die natürliche Gränzen der Residenz, oder wo solche nicht bestimmt genug seynd,

24 Sänger (wie Anm.13) S. 197.

${ }^{25}$ Haberkern/Wallach (wie Anm.11), Bd.1, S. 90. Selbst Margret Sänger definierte die Burgfriedensbegrenzung lediglich wie folgt: „Die Burgfriedensbegrenzung setzt in der Regel an einem Punkt, einer Brücke, einer Mühle, einem Weg, einer Furt usw. ein, umreißt dann das Gebiet und endet wieder am Ausgangspunkt“; SÄNGER (wie Anm. 13) S. 200. Vgl. auch Art. Burgfriede, in: Allgemeine Encyklopädie der Wissenschaften und Künste, Leipzig $1818-1889$, Bd.14, S.66-67.

26 S. Maurer (wie Anm. 2) S. 105 und Margret Lemberg, Juliane Landgräfin zu Hessen (1587-1643). Eine Kasseler und Rotenburger Fürstin aus dem Hause Nassau-Dillenburg in ihrer Zeit (Quellen und Forschungen zur hessischen Geschichte, Bd. 90), Darmstadt/Marburg 1994, S. 126.

27 Rapp (wie Anm. 3) S. 243.

28 Patze (wie Anm.3) S.543. Vgl. auch Sabine M. Weigand-Karg, Die Plassenburg. Residenzfunktion und Hofleben bis 1604, Bayreuth 1991, S.224f.

29 Patze (wie Anm. 3) S. 552. Zur Größe des Burgfriedensbezirks in Würzburg s. Gerda Zimmermann, Der Hofstaat der Fürstbischöfe von Würzburg von 1648 bis 1803. Verfassung und Entwicklungsgeschichte, Diss. Jur., Würzburg 1976, S. 47.

30 Moser (wie Anm. 4) Bd.2, S. 800. 


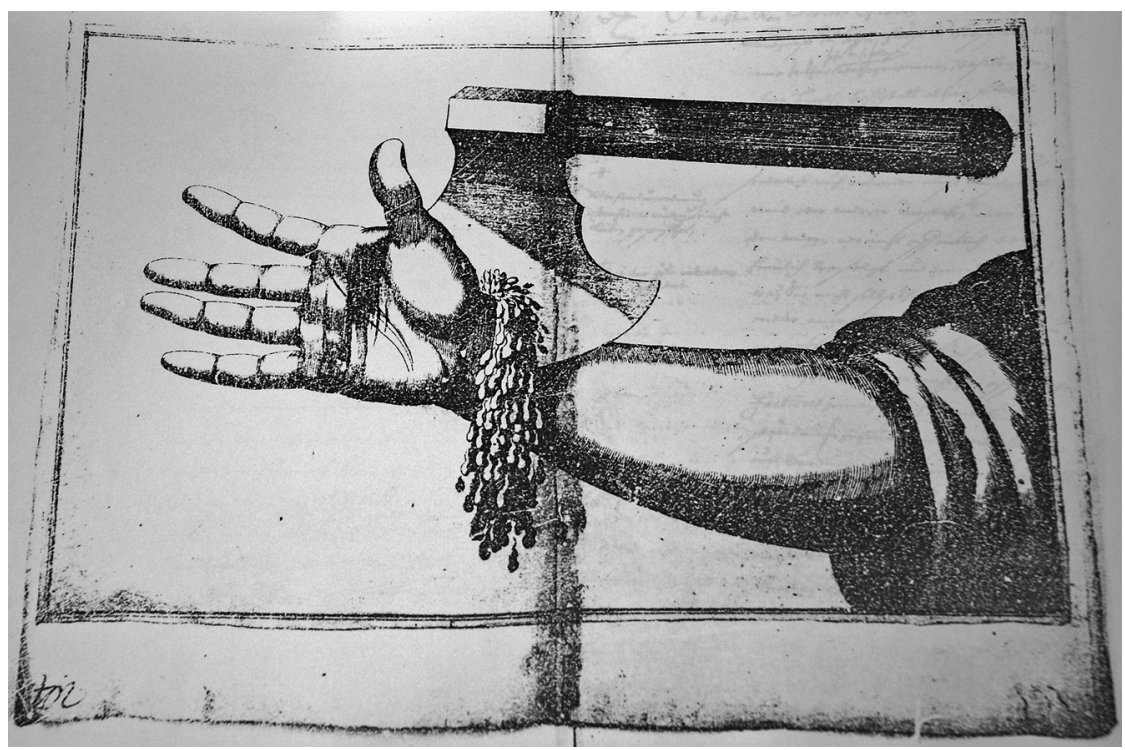

Abb. 1: Strafe des Handabschlagens (nach Landgraf Georg II. Hessen, Burgfriedensordnung Hessen-Darmstadt 1624/1627, StA Darmstadt, Hausarchiv, Abt. 4, Konv. 188, Fasc. 4).

dienen gewisse Steine, Crentze etc. zu Merckmablen. Wo der Burg-Fride einen weitern Umfang hat und auch privat Gebäude in sich faßt, seynd hie und da Pfäblen mit einer Tafel eingeschlagen, auf welchen eine auf einen Block liegende und durch ein darüber schwebendes Beil abgehauene Hand gemablt ist, mit der Beyschrift Burg-Fride. [...] Der Burg-Fride erstreckt sich aber auch auf alle andere LandLust- und Jagd-Häuser, auch alle übrige Orte, wo sich der Regent selbst gegenwärtig befindet ${ }^{31}$ (Abb.1).

Soweit zur Bedeutung der Begriffe „Burgfrieden“ und „Burgfriedensbezirk“ im Spätmittelalter. Wie wurde der Begriff dann in der Frühen Neuzeit benutzt bzw. meint der Burgfrieden in den württembergischen Hofordnungen des beginnende 17. Jahrhunderts noch das gleiche wie im Spätmittelalter?

Auf den zweiten Teil der Frage gibt es eine einfache Antwort: Nein. Wie bereits in der zu Beginn angeführten Definition aus dem „Deutschen Rechtswörterbuch“ deutlich wurde, unterliegt der Burgfrieden einem Bedeutungswandel. Inwieweit sich die frühneuzeitliche Definition aus der spätmittelalterlichen ableiten lässt, oder ob sie quasi neu entstand, bedürfte einer gründlichen und eingehenden Untersuchung, die mit diesem Beitrag leider nicht geleistet werden kann.

31 Ebd., Bd.2, S. 800. 
Klar ist jedoch, dass sich die Forschung mit der Bedeutung und Definition des Burgfriedens in der Frühen Neuzeit bisher kaum auseinandergesetzt hat. Eine erste Idee findet sich in der „Allgemeinen Encyklopädie der Wissenschaften und Künste“, herausgegeben von Johann Samuel Ersch und Johann Gottfried Gruber $^{32}$. In dieser Enzyklopädie findet sich als vierte Definition folgende: Noch ist einer erst später aufgekommenen Bedeutung des Worts Burgfriede zu gedenken, welche eigentlich nur auf Burgen und Schlösser der mit Landeshobeit begabten Reichsstände Beziehung [hat]. Seitdem diese anfingen, ibr, Hofgesinde - nach heutigem Sprachgebrauch ibren Hofstaat, zu vermebren, [...] und die Großen sich häufger als früber ständige Wohnsitze, Residenzen, wählten, ward an den meisten Höfen nöthig gefunden, eigene Polizeiordnungen und Vorschriften, hauptsächlich zur Erhaltung der Rube und Sicherbeit im Schloß und dessen Bezirk aufzustellen zu lassen. Darin waren dann auch besondere Strafen für die Übertreter und Unruhestifter festgesetzt, gewöhnlich weit härter, als die sonst üblichen [...] weil jedes Vergeben in der Nähe und in der Wobnung des Herrn zugleich für eine diesem selbst zugefügte Beleidigung geachtet ward. Dergleichen Hausordnungen wurden, auch ganz angemessen, Burgfrieden genannt [...]. “33

Eben diese von Ersch und Gruber hier angeführte Bedeutung ist auch jene, die die Basis für Maurer gebildet hatte, der ebenfalls für die Zeit seit etwa dem 16. Jahrhundert einen solchen Bedeutungswandel in den Quellen aufgezeigt hatte ${ }^{34}$.

Bereits im Mittelalter hatten auf den mit Burgfrieden begabten Burgen besondere Verhaltensregeln und besondere "Gesetze“ gegolten, sogenannte borchvredes rechte ${ }^{35}$, und spätestens seit dem ausgehenden 14 . und dem 15 . Jahrhundert wurden auch explizit Strafen für verschiedene Vergehen genannt ${ }^{36}$, aber, so bemerkte Rapp: „Vergleicht man die verschiedenen Burgfriedensbestimmungen untereinander, so fällt vor allen Dingen auf, daß sich ihr Text im Laufe der Zeit ständig verlängert hat. [...] Die Klauseln vermehrten sich; präzis wurden sie sozusagen ausgeklügelt [...]. “37 Zudem wurden spätestens seit Beginn des 15. Jahrhunderts nicht mehr nur die Ganerben auf den Burgfrieden und die in ihm enthaltenen Vorschriften verpflichtet, sondern auch die Dienerschaft, die sich, wenn auch nur zeitweise, auf der Burg befand ${ }^{38}$.

Diese ersten bekannten Friedensregelungen sind nicht besonders ausgefeilt, sondern denkbar einfacher Natur; so steht etwa in der Burgfriedensordnung der Plassenburg aus dem Jahr 1547 schlicht, das jeder, so sich in berürtem Haus Blas-

\footnotetext{
32 Allgemeine Encyklopädie der Wissenschaften und Künste, 167 Bde., Leipzig 18181889.

33 Ebd., S. 67.

4 Maurer (wie Anm. 2) S. 105.

S. Patze (wie Anm. 3) S. 546.

36 S. ebd., S. 548.

37 Rapp (wie Anm.3) S. 248.

38 S. ebd., hier S. 244.
} 
senburg und derselben erstreckten Burckfriedens orth mit einen andern sich hadern und in unwillen einzulassen, ein webr gethon um ein Handt und der so den straich verbracht um das Haubt gestrafft wird $^{39}$.

Betrachtet man die angeführten Strafen, vor allem die des Handabschlagens, so muss man davon ausgehen, dass die Basis dieser Burgfriedensregelungen im militärischen Bereich zu suchen ist, denn nur hier finden sich vergleichbar harte Strafen für eigentlich eher kleine Vergehen ${ }^{40}$. Maurer allerdings wies darauf hin, dass diese scharfen Strafen im Mittelalter nicht nachzuweisen sind, dort sei in den Bestimmungen nur von „Bußen, Sühne und von Ausweisung der Schuldigen die Rede.“41 Er schloss daraus, dass sich eben dieses scharfe Strafrecht, das uns spätestens seit dem 16. Jahrhundert auf nahezu allen Festungen und Schlössern begegnet, aus einer anderen Quelle entwickelt haben muss. Ebenso sah dies Weigand-Karg für den Raum Brandenburg-Ansbach. Beide kamen zu dem Schluss, dass einzig eine Herleitung aus dem Militärrecht möglich sei und Sinn mache ${ }^{42}$. Maurer sah das „unmittelbare Vorbild der württembergischen Festungsordnungen“ im „Artikelbrief des Schwäbischen Bundes für den Feldzug gegen den Herzog Ulrich von Württemberg im Jahre 1519“ und ging davon aus, dass „einzelne Bestimmungen des Kriegsrechts [...] auf den Festungen des 16. Jahrhundert[s] zu Dauerrecht" wurden ${ }^{43}$.

Dass diese Herleitung wohl auch tatsächlich so gesehen werden muss, wird an den Plassenburger Ordnungen deutlich, denn in der Zeit, in der vor allem militärische Notwendigkeiten im Vordergrund standen, waren die Strafen extrem hart und dienten primär der allgemeinen Disziplinierung, vor allem der Vermeidung von Tätlichkeiten und körperlichen Auseinandersetzungen. In späterer Zeit jedoch, genauer gesagt in der Ordnung von 1603, treten plötzlich andere Aspekte in den Vordergrund: Die Plassenburg war nun eher Residenz denn Burg und die neue Burgfriedensordnung war deutlich detaillierter geworden und vor allem eher auf höfische Repräsentation ausgelegt. Moralische Maßstäbe werden wichtiger, der Besuch der Gottesdienste nimmt einen breiten Raum ein, und die Dienerschaft soll erbar, uffrichtig, eingetzogen, bescheiden, sittsam, friedlich, fromm und gottesfürchtig $\operatorname{sein}^{44}$. Außerdem werden die Strafen nun deutlich „humaner“, und

39 Zitiert nach Weigand-Karg (wie Anm. 28) S. 225. Vgl. auch Veit Ludwig von SeckenDORFF, Teutscher Fürsten-Staat, Jena 1737, ND Aalen 1972, S. 575.

40 Bereits Moser sah diesen Zusammenhang von Militärrecht und Burgfrieden, s. Moser (wie Anm. 4), Bd. 2, S. 795 f.

${ }^{41}$ Maurer (wie Anm. 2) S. $114 \mathrm{f}$.

42 S. Weigand-Karg (wie Anm. 28) S. 225 f. und Maurer (wie Anm. 2) S. 114, der gerade das Handabschlagen bis auf die Heeresordnung Barbarossas zurückführt. Auch in Würzburg waren Strafen wie das Abschlagen der Hand bis ins 17. Jahrhundert hinein laut Hofordnung bzw. Burgfrieden vorgesehen, s. Zimmermann (wie Anm.29) S. 47.

${ }^{43}$ Maurer (wie Anm. 2), hier S. 115.

44 Zitiert nach Weigand-Karg (wie Anm. 28) S. 226. 
Manches war bereits mit einer kleinen Spende in die Almosenkasse aus der Welt zu schaffen. Die harten Strafen, die knapp 60 Jahre zuvor ausgesprochen wurden, sollten aber für schwere Vergehen durchaus weiter bestehen bleiben.

Fritz Hasenritter wies für die Pommerschen Hofordnung letztlich das gleiche Phänomen nach: Auch dort wurde „eine besondere Bestimmung über den Burgfrieden [...] seit 1560 gebräuchlich. " ${ }^{45}$ Allerdings wies Hasenritter auch darauf hin, dass diese scharfen Strafbestimmungen anscheinend wenig Wirkung zeitigten, denn ein Begleitschreiben Herzog Philipps II. von Pommern zur Hofordnung des Jahres 1616 zeigt deutlich seinen Ärger über zahlreiche Schlägereien, Trinkgelage sowie Mord und Totschlag bei Hof, die aber nicht wirklich geahndet würden ${ }^{46}$.

Sicherlich war es auch notwendig, die einstmals aus dem militärischen Bereich übernommenen harten Strafen abzumildern und zu verändern, denn insbesondere kleinere körperliche Auseinandersetzungen waren an den Höfen der Frühen Neuzeit anscheinend an der Tagesordnung. Nicht umsonst galt Deutschland mit seinen Höfen in diesem Zeitalter als ein „Hort des Grobianismus“47. So kam auch Wolfgang Ludwig Assum, Hofprediger des Grafen von Hohenlohe-Weikersheim, zu der Auffassung, dass man die körperlichen Strafen, wie sie der Burgfrieden enthält, wohl nicht in dieser Form anwenden solle, denn wann man nach der Schärffe des verpeenten Burgfriedens allen solchen Balgern sollte die Finger spitzen / und die Händ abhauen / so mōchte der Hofstatt besser einem Lazarethhaus / als einem Königlichen Hofläger kōnnen verglichen werden. ${ }^{48}$

Dass die Zustände an deutschen Höfen in der Tat oftmals beklagenswert waren und es regelmäßig zu Straftaten unterschiedlichster Art kam, verdeutlicht auch ein ironisch verfasster Neujahrsgruß des Kölner Erzbischofs und Kurfürsten Joseph Clemens, der von Braubach wiedergegeben wurde: „In die Kammer gehöre ein Maulkorb, ,damit daraus selbe nicht schwätzen können', [...] in den Keller ,ein Schloß, daß er allezeit versperrt bleibe und nicht von jedermann geöffnet werde‘, in die Küche ein langer Mantel, ,darunter selbe das Austragen besser verbergen können' $[\ldots]$ endlich die ,anderen gemeinen Hofweiber von der dritten Klasse mit

45 Fritz Hasenritter, Die pommerschen Hofordnungen als Quellen für die Hof- und Landesverwaltung, in: Baltische Studien (1937) S. 147-182, hier S. 153.

46 S. ebd.

47 S. Georg Lauterbeck, Regentenbuch, Leipzig 1557, Buch 2, Kap. 7. Ähnlich auch das von Hasenritter zitierte Begleitschreiben Herzog Philipps von Pommern zur Hofordnung von 1616, in dem er beklagte, dass es aufgrund des hohen Alkoholkonsums ständig zu Mord und Todschlag käme, s. HasenritTer (wie Anm.46) S. 153.

48 Wolfgang Ludwig Assum, Kirchen Regiment= und Hauß=Spiegel. In welchem Aus dem Canonischen Buch Esther/Der hochbedrängten aber nicht übermochten Kirchen Gottes Eingentliches Contrafayr/So dann Weltlicher Potentaten Regierungs und Hofstaats Modell/In zweyen unterschiedenen Theilen deutlich für Augen gestellet wird, Nürnberg 1672, Teil II, S. 153. 
der Tafel Moses, damit sie die zehn Gebote Gottes besser in Obacht nähmen. Allen Hofkindern droht eine gute Rute $[\ldots]^{\text {‘. }}$ “49

Die Überwachung des Burgfriedens und die Strafgewalt oblagen in erster Linie dem Inhaber des obersten Hofamts, damit in vielen Fällen dem Marschall bzw. Hofmarschall ${ }^{50}$. In Württemberg tritt in dieser Position zunächst der Haushofmeister in Erscheinung, der zum einen das Gesinde annahm und zum anderen über die Einhaltung des Burgfriedens wachte ${ }^{51}$.

Wer sich also gegen den Burgfrieden verging, der wurde in aller Regel zunächst verhaftet, in Verwahrung genommen und dann dem obersten Hofbeamten vorgeführt. In schwerwiegenden Fällen wurde häufig noch der Regent in Kenntnis gesetzt, der dann mit über die entsprechende Strafe entschied, erst danach wurde der Delinquent al $\beta$ hal $\beta$ umb hal $\beta$, handt umb handt gestraft ${ }^{52}$.

\section{Hofordnungen}

Das soziale Gebilde Hof, mit seinen zum Teil hunderten und mehr Mitgliedern bedurfte unzweifelhaft einer Ordnung, allein schon, um den Alltag organisieren, Auseinandersetzungen und Differenzen beilegen zu können und die Hofmitglieder zu disziplinieren. Diese Ordnungen wurden wahlweise mündlich tradiert oder auch schriftlich festgehalten ${ }^{53}$. In späterer Zeit scheint es zudem so zu sein, dass derartige Ordnungen notwendig wurden, um die Standesmäßigkeit eines

49 Zitiert nach Max Braubach, Die vier letzten Kurfürsten von Köln. Ein Bild Rheinischer Kultur im 18. Jahrhundert, Bonn/Köln 1931, S.21f.

50 S. Treusch von Buttlar (wie Anm. 4) S. 14; Ivan Ritter von Zolger, Der Hofstaat des Hauses Österreich (Wiener Staatswissenschaftliche Studien, Bd.14), Wien/Leipzig 1917, S. 104-117; Zimmermann (wie Anm. 29) S. 40 und S.46f.; Karin Plodeck, Hofstruktur und Hofzeremoniell in Brandenburg-Ansbach vom 16. bis zum 18. Jahrhundert. Zur Rolle des Herrschaftskultes im absolutistischen Gesellschafts- und Herrschaftssystem, Ansbach 1972, S. 96 f. und S. 101. Friedrich Karl von Moser dehnte diese Befugnisse aus und schrieb, dass die Bewahrung des Burgfriedens im Grunde allen Hofbedienten obliege, in Sonderheit aber dem Hofmarschall, Schlosshauptmann und Burggrafen, s. Moser (wie Anm.4), Bd.2, S. 801.

51 S. Kern (wie Anm. 7), Bd.1, S. VIII.

52 Zitiert nach Zimmermann (wie Anm. 29) S. 48.

${ }^{53}$ Zur mündlichen Tradition von höfischen Ordnungen s. Detlev KraAk, Höfe und Hofordnungen. Kolloquiumsbericht, in: Mitteilungen der Residenzen-Kommission der Akademie der Wissenschaften zu Göttingen (1996) S.17-26, Stellungnahme des Veranstalters, S.22f. und die dort angesprochenen Artikel von Peter Moraw, Zusammenfassung, in: Höfe und Hofordnungen (wie Anm. 8) S. 555-560; Michail A. Bojcov, Sitten und Verhaltensnormen am Innsbrucker Hof des 15. Jahrhunderts im Spiegel der Hofordnungen, ebd., S. 243-283; Paul Joachim Heinig, Theorie und Praxis der ,höfischen Ordnung' unter Friedrich III. und Maximilian I., ebd., S. 223-242, und Hartmut Boockmann, Hof und Hofordnung im Briefwechsel des Albrecht Achilles von Brandenburg, ebd., S.315-320. 
Hofes zu symbolisieren. Doch wie und aus welcher Basis entwickelten sich diese Ordnungen des Hofes und wie sahen sie konkret aus?

Für den späteren deutschen Sprachraum existieren vor dem 13. Jahrhundert nur wenige Schriften, die Auskunft über die Ordnung des Hofes geben; eine davon ist die De ordine palatii Hinkmars von Reims aus dem Jahr $882^{54}$. Hier werden die am karolingischen Hof bestehenden Ämter und deren Aufgaben detailliert dargestellt. Allerdings ist dieses Werk wohl weniger als Hofordnung denn als theoretisches Werk über die Ordnung des Hofes anzusehen. Für das Karolingische und Deutsche Reich blieb dieses Werk Hinkmars lange Zeit einzigartig.

Anders sieht es im byzantinischen Machtbereich aus. Hier ist vor allem der Liber de Ceremoniis Kaiser Konstantins VII. hervorzuheben, der nicht nur die Ämter am Hof, sondern auch das höfische Zeremoniell genau beschreibt ${ }^{55}$. Auch in England finden sich ab 1135 solche Beschreibungen des Hofes ${ }^{56}$ und vor allem in Frankreich wurden seit dem 13. Jahrhundert regelmäßig Hofordnungen verfasst ${ }^{57}$. Auch für den Hennegau liegt mit der Ministeria curie Hanoniensis von 1212/14 eine frühe Ordnung des Hofes vor, die vor allem ein ausführliches Hofämterverzeichnis darstellt ${ }^{58}$.

In Spanien finden sich seit dem ausgehenden 13. und beginnenden 14. Jahrhundert Hofordnungen. $\mathrm{Zu}$ nennen sind vor allem die Leges Palatinae Jakobs II. von Mallorca ${ }^{59}$, die einen neuen Abschnitt in der Geschichte der europäischen

${ }^{54}$ Hinkmar von Reims, De Ordine Palatii, hg. von Thomas Gross/Rudolf Schieffer (MGH Fontes Iuris Germanici Antiqui, Bd.3), Hannover 1980.

55 S. Karl Arnold Willemsen, Zur Genesis mittelalterlicher Hofordnungen, in: Personalund Vorlesungsverzeichnis der Staatlichen Akademie zu Braunsberg, 2. Trimester, Gumbinnen 1940, S.7.

56 S. etwa die Constitutio domus Regis aus dem Jahr 1135 und die im Jahr 1318 entstandene Hofordnung Eduards II. aus York, ebd., S.7-12; Thomas ZотZ, Hof und Hofordnung vor der Zeit der Verschriftlichung, in: Höfe und Hofordnungen (wie Anm. 8) S. 65-73, hier S.69, und Arnd Reitemeier, Die Ordnung des englischen Hofes um 1400 - eine Ordnung nach Wissen?, ebd., S.117-140.

$57 \mathrm{Zu}$ nennen sind hier vor allem die 1261 entstandene Hofordnung Ludwigs des Heiligen und die aus dem Jahr 1285 stammende Hofordnung Philipps III., s. Willemsen (wie Anm.56); K.-H Ahrens, Art. Hofordnung, in: LexMA 5, Sp.74-76, hier Sp.75, und Christina Hofmann-Randall, Die Herkunft und Tradierung des Burgundischen Hofzeremoniells, in: Jörg Jochen BERNs/Thomas RAHN (Hg.), Zeremoniell als höfische Ästhetik in Spätmittelalter und Früher Neuzeit, Tübingen 1995, S. 150-156, hier S. 150-153.

58 S. Giselbert von Mons, Chronicon Hanoniense, hg. von Georg Heinrich PerTz (MGH Scriptores, Bd.21), Hannover 1869, S. 481-601.

59 Die „Leges palatinae“ entstanden im Jahr 1337, s. Willemsen (wie Anm. 55) S. 20-23. Zum mallorquinischen Hof s. Gottfried Kerscher, Die Strukturierung des mallorquinischen Hofes um 1330 und der Habitus der Hofgesellschaft, in: Höfe und Hofordnungen (wie Anm. 8), S.77-89. Zu den aragonischen Hofordnungen s. Karl SchwarZ, Aragonische Hofordnungen im 13. und 14. Jahrhundert. Studien zur Geschichte der Hofämter und Zentralbehörden des Königreichs Aragon (Abhandlungen zur Mittleren und Neueren Geschichte, Heft 54), Berlin/Leipzig 1914. 
Hofordnungen darstellen, da ihr Inhalt verglichen mit den Vorgängerschriften deutlich erweitert ist. Zu den bis zu diesem Zeitpunkt typischen Ämteraufstellungen und Gehaltslisten treten hier Anweisungen über das Verhalten der Amtsinhaber und Hofbediensteten. Hinzu kommt, dass die Leges als einzige bekannte Hofordnung nicht in Form einer simplen Kanzleihandschrift vorliegen, sondern als Prachthandschrift ${ }^{60}$.

Am bekanntesten und am besten erforscht sind sicherlich die burgundischen Hofordnungen, die als Paradebeispiele mittelalterlicher Hofordnungen gelten. Nirgends sonst sind Hofordnungen in einem derartigen Maße und in solcher Vollständigkeit überliefert ${ }^{61}$. Diese Ordonnances de l'Hôtel waren in erster Linie Amtsträgerlisten, die sowohl die Amtszeit als auch die Gagen der Amtsinhaber verzeichneten $^{62}$, wohingegen sich Dienstanweisungen eher selten finden. Erst in der Zeit Karls des Kühnen, also um das Jahr 1469, änderte sich das Aussehen, und es traten zunehmend zeremonielle und disziplinarische Inhalte in den Ordnungen auf 63 .

Die ältesten im Deutschen Reich überlieferten Hofordnungen stammen aus den Jahren 1293 und 1294 und werden auch als Vilshofener Vertrag bezeichnet ${ }^{64}$. Bischof Heinrich von Regensburg und Graf Gebhard von Hirschberg arbeiteten diese Ordnungen gemeinsam mit den Räten der Herzogsbrüder Otto III., Ludwig III. und Stephan I. im Rahmen eines Teilungsvertrages aus ${ }^{65}$.

Die Inhalte dieser frühen Ordnungen für den Hof sind noch sehr unterschiedlich. „In formaler Hinsicht reicht die Palette von kurzen listenartigen Aufstellungen bis hin zu umfassenden Regulativkonvoluten mit zum Teil detaillierten Beschreibungen von Amtsbereichen und einzelnen Dienstvorschriften (Aragón um 1300; »Leges Palatinae«, Mallorca 1337; Dauphiné 1340; Burgund 1468/69; »Black Book«, England 1471/72). “66

Die ältesten dieser Ordnungen sind meist reine Personalaufstellungen mit wenigen Dienstanweisungen, wobei auch nur die höheren Hofangestellten Beachtung

60 Ebd.

61 S. Holger Kruse, Hof, Amt und Gagen. Die täglichen Gagenlisten des burgundischen Hofes (1430-1467) und der erste Hofstaat Karls des Kühnen (Pariser Historische Studien, Bd.44), Bonn 1996; Kruse (wie Anm. 8) und Kruse/Paravicini (wie Anm. 8).

62 S. E. Lalou, Art. Ordonnances de l'Hôtel, in: LexMA 6, Sp. 1442-1443, und Elisabeth LALou, Les ordonnances de l'hôtel des derniers Capétiens directs, in: Höfe und Hofordnungen (wie Anm. 8) S. 91-101.

63 S. KraAK (wie Anm.54) S.20f. und Jacques Paviot, Ordonnances de l'hôtel et cérémonial de cour aux $\mathrm{XV}^{\mathrm{e}}$ et $\mathrm{XVI}^{\mathrm{e}}$ siècles, d'après l'exemple bourguignon, in: Höfe und Hofordnungen (wie Anm. 8) S.167-174.

64 S. Wilhelm STörmer, Hof und Hofordnung in Bayern-München (15. und frühes 16. Jahrhundert), ebd., S.361-381, hier S. 363.

65 S. Karl-Ludwig Ay, Altbayern von 1180 bis 1550 (Dokumente zur Geschichte von Staat und Gesellschaft in Bayern, Bd. I/2), München 1977, S. 596.

66 Ahrens (wie Anm.57) Sp. 75 f. 
fanden. Zudem finden sich häufig detaillierte Listen mit den am Hof befindlichen Pferden ${ }^{67}$. Diese Listen finden sich in späterer Zeit nicht mehr, vor allem, da der militärische Aspekt der Ämter in den Hintergrund trat bzw. völlig verschwand. Zudem wurden die Höfe sesshaft, Residenzen entstanden, so dass die Amtsinhaber keiner eigenen Pferde mehr bedurften, um von einem Hofort zum anderen zu ziehen.

Stattdessen trat nun der Ordnungsaspekt in den Vordergrund, und Anweisungen über das christliche Leben und den alltäglichen Lebensstil am Hof füllten weite Teile der Hofordnungen, wie bereits zuvor gesehen. Auch zeremonielle Aspekte nahmen spätestens ab dem 17. Jahrhundert in den Hofordnungen immer breiteren Raum ein, was besonders an den bayerischen Hof- und Kammerordnungen Karl Theodors und Maximilian Josephs aus den Jahren 1794 und 1800 auffällt, die im Grunde reine Zeremonialvorschriften sind ${ }^{68}$.

Diese knappen Ausführungen zeigen bereits, dass Hofordnungen als Quellen lange nicht so eindimensional sind, wie ihnen die Forschung häufig nachgesagt hat ${ }^{69}$. Nicht nur, dass sie sich in formaler Hinsicht vielfach deutlich voneinander unterscheiden, auch ihre Inhalte differieren stark ${ }^{70}$. So schrieb Holger Kruse völlig zutreffend: „Wir verwenden den Begriff ,Hofordnung“, nicht weil er der exakteste wäre, sondern weil er der heute gebräuchliche ist“, denn „schon von Zeitgenossen [wurde] eine ganze Reihe verschiedener Dokumente [...] als ,Hofordnungen` bezeichnet $[\ldots]$. “71

Diese Vielfältigkeit der als „Hofordnung“ bezeichneten Quellen machte es notwendig, sie in verschiedene Typen zu unterteilen: Brigitte Streich schlug drei Typen von Hofordnungen vor:

67 Bojcov (wie Anm. 53) S. 245 und Flink/Thissen (wie Anm. 9).

68 Churfürst Karl Theodor, Churpfalzbaierische Hof- und Kammerordnung. 6. Brachmonats (Juni) 1794, verfügbar unter http://www.mdz-nbn-resolving.de/urn/resolver. pl?urn=urn:nbn:de:bvb:12-bsb10319835-5 (Zugriff: 27.08.2014); Churfürst Maximilian JosepH, Churpfalzbaierische Hof- und Kammerordnung. 2. Jäner 1800, verfügbar unter http://bavarica.digitale-sammlungen.de/resolve/display/bsb10319880.html (Zugriff: 10.09. 2014).

69 S. Michael Reinbold, Hof und Landesverwaltung in Dannenberg 1570-1636. Hofund Kanzleiordnungen als Spiegel herrscherlichen Selbstverständnisses am Beispiel einer welfischen Sekundogenitur, in: Niedersächsisches Jahrbuch für Landesgeschichte (1992) S. 53-70, hier S. 69f.; Rainer A. Müller, Der Fürstenhof in der Frühen Neuzeit (Enzyklopädie Deutscher Geschichte, Bd.33), München 1995, S. 40 f.

70 S. Anja Kircher-Kannemann, Heilsame auffsicht und verfassung. Hofordnungen vom Mittelalter bis zur Neuzeit, Diss. phil., Düsseldorf 2015, verfügbar unter: http://docserv.uni-duesseldorf.de/servlets/DerivateServlet/Derivate-37296/Heilsame\%20auffsicht\%20und\%20verfassung.pdf (Zugriff: 01.04.2016), S.173-216 und KraAK (wie Anm.54) S.18, der daher vorschlug, lieber von „Ordnungen am Hof“ zu sprechen.

71 Kruse (wie Anm. 8) S. 143. 
1. Ordnungen, die aus Gründen der Kostenreduktion entstanden sind, wobei dieser Typ ihrer Ansicht nach am häufigsten vorkommt ${ }^{72}$,

2. Ordnungen, die aufgrund von Personalwechseln entstanden und der Organisation der $\ddot{\text { Amter dienten }}{ }^{73}$ und

3. Umfassende „Regimentsordnungen“, die auf Druck der Stände oder des Rates bei Herrscherwechsel oder im Rahmen von Steuerbewilligungen erlassen wurden ${ }^{74}$.

Aber es gibt noch zahlreiche andere Definitionsversuche im Zusammenhang mit Hofordnungen. Nur einige wenige sollen hier kurz erwähnt werden: Auge und Spiess etwa sehen in Hofordnungen den Ausdruck fürstlicher Herrschaftsansprüche und das Verlangen des Fürsten eine feste Bindung zwischen Herrscher und Hof $\mathrm{zu}$ erreichen ${ }^{75}$. Vec wiederum sieht in ihnen zum einen Finanzdokumente, aber auch einen Ausdruck der „Guten Ordnung“ und damit eine Form der Policeyordnung ${ }^{76}$. Ihr Inhalt sei ein Zeichen der „binnenhöfischen Totaldisziplinierung "77. Es finden sich tatsächlich in einigen Hofordnungen Zitate aus Policeyordnungen, was die enge Verwandtschaft dieser beiden Quellengattungen belegt ${ }^{78}$.

Willoweit sieht in Hofordnungen ein Zeichen der allgemeinen „Regelungswut“ und der Sozialdisziplinierung ${ }^{79}$. Außerdem wies er darauf hin, dass sie zudem „die

72 Brigitte STREICH, Die Diepholzer Hof- und Verwaltungsordnungen des 16. Jahrhunderts. Zur Anwendung und Fortschreibung eines Herrschaftsregulativs, in: Höfe und Hofordnungen (wie Anm. 8) S. 497-518, hier S. $497 \mathrm{ff}$.

73 Vgl. Reinbold (wie Anm. 69) und Plodeck (wie Anm. 50) S. $34 \mathrm{ff}$.

74 STREich (wie Anm.72) S.498. Streich schneidet hier tatsächlich ein Definitionsproblem an, denn es ist die Frage, ob Regimentsordnungen als Hofordnungen anzusehen sind, vgl. Bouterwek, Die Regiments-Ordnung vom 11. Dezember 1592, in: Zeitschrift des Bergischen Geschichtsvereins (1865) S.212-243. In dieser Regimentsordnung steht ausdrücklich, dass die Hofordnung neben der Regimentsordnung unverändert weiter Bestand haben solle.

75 Oliver Auge/Karl-Heinz Spiess, Hof und Herrscher, in: Werner Paravicini (Hg.), Höfe und Residenzen im spätmittelalterlichen Reich. Bilder und Begriffe (Residenzenforschung, Bd.15.II), Ostfildern 2005, S.3-15, hier S. 4.

${ }^{76}$ Miloš Vec, Hofordnungen. Versuch einer rechtshistorischen Funktionsanalyse. Zu einem Beispiel spätmittelalterlicher Normsetzung, in: Höfe und Hofordnungen (wie Anm. 8) S.43-63, hier S.44f. Vgl. auch Plodeck (wie Anm.50) S.238ff. und Wolfgang Wüst, Hof und Policey. Deutsche Hofordnungen als Medien politisch-kulturellen Normenaustausches vom 15. bis zum 17. Jahrhundert, in: Werner PARAvicini/Jörg WeTtLaufer (Hg.), Vorbild - Austausch - Konkurrenz. Höfe und Residenzen in der gegenseitigen Wahrnehmung. 11. Symposium der Residenzen-Kommission der Akademie der Wissenschaften zu Göttingen (Residenzenforschung, Bd.23), Wien, 20.-24. September 2008, Ostfildern 2010, S. 115-134, hier S. 115.

77 Vec (wie Anm. 76) S. 47. Vgl. hierzu auch WüHrer/Scheutz (wie Anm. 9) S. 27.

78 S. Wüst (wie Anm.76), hier S.116.

79 Dietmar Willoweit, Hofordnungen als Zeugnisse des Rechtsdenkens, in: Reinhardt Butz/Jan Hirschbiegel/Dietmar Willoweit (Hg.), Hof und Theorie. Annäherungen an ein historisches Phänomen (Norm und Struktur, Bd.22), Köln 2004, S. 165-178, hier S. 169. 
Kontinuität der Hofkultur“ abbildeten, denn es gäbe sie während der gesamten Zeit der höfischen Geschichte ${ }^{80}$. Hesse hingegen sieht diese Quellen als Teil des frühneuzeitlichen Modernisierungsprozesses und als Mittel zur Beschränkung der fürstlichen Herrschaft ${ }^{81}$. Diesen Thesen setzte Sommer entgegen, dass Hofordnungen vor allem dazu dienten, den Alltag zu organisieren und die Hofpolicey zu erhalten ${ }^{82}$. Als Quellen des höfischen Alltags und dessen Organisation sehen auch Kern und Paravicini Hofordnungen $\mathrm{an}^{83}$. Flink hingegen sieht vor allem in den klevischen Hofordnungen weder Finanz- noch Zeremonialordnungen, sondern einen Teil der „Ökonomie“ des Hofhalts, wobei zu beachten sei, dass der frühneuzeitliche „Oeconomiebegriff“ weit über den Bereich der Finanzen hinausgriff ${ }^{84}$.

Ellen Widder erweiterte all diese Definitionen um einen interessanten und bis zu diesem Zeitpunkt vernachlässigten Aspekt, denn ihrer Ansicht nach waren Hofordnungen vor allem ein Ausdruck des standesgemäßen Hofes. Sie schreibt ihnen einen symbolischen Charakter zu, da ein Hof ohne Hofordnung Gefahr laufe sozial abzusteigen ${ }^{85}$.

Vgl. auch Paul MüNCH, Das Jahrhundert des Zwiespalts. Deutsche Geschichte 1600-1700, Stuttgart 1999, S. 90 und Heinig (wie Anm. 54) S.335f.

80 Willoweit (wie Anm.79) S. $175 \mathrm{f}$.

81 S. Christian Hesse, Zwischen Reform und Beschränkung. Die Hofordnungen der Landgrafschaft Hessen aus dem beginnenden 16. Jahrhundert, in: Höfe und Hofordnungen (wie Anm. 8) S.337-360, hier S.337ff.

82 S. Dagmar Sommer, Hofordnungen. Ordnung ist die Seele des Hofes, in: Jörg Jochen Berns (Hg.), Erdengötter. Fürst und Hofstaat in der Frühen Neuzeit im Spiegel der Marburger Bibliotheks- und Archivbestände (Schriften der Universitätsbibliothek Marburg, Bd.77), Marburg 1997, S.73-89, hier S. 73 f. Vgl. Max Hein, Preußische Hofordnungen des 16. Jahrhunderts, in: Altpreußische Forschungen (1925) S. 52-68, hier S. 52.

83 S. Kern (wie Anm.7), Bd.1, S. IX; Werner Paravicini, Alltag bei Hofe, in: Werner Paravicini (Hg.), Alltag bei Hofe. 3. Symposium der Residenzen-Kommission der Akademie der Wissenschaften in Göttingen, Ansbach, 28. Februar bis 1. März 1992 (Symposium der Residenz-Kommission der Akademie der Wissenschaften in Göttingen, Bd.3), Sigmaringen 1995, S. 9-30, hier S. 10.

${ }^{84}$ S. Klaus Flink, Die klevischen Hofordnungen. Von der Kostliste bis zur Regiments-Ordnung, in: Höfe und Hofordnungen (wie Anm. 8) S.401-420, hier S.418. Vgl. auch Volker BAUER, Hofökonomie. Der Diskurs über den Fürstenhof in Zeremonialwissenschaft, Hausväterliteratur und Kameralismus (Frühneuzeitstudien, NF Bd.1), Köln/Weimar/Wien 1997; Klaus Neitmann, Fürst und Räte vor der Herausforderung, guter Ökonomie und Haushaltung'. Aufbau und Unterhaltung der Hof- und Landesverwaltung des erzbischöflich rigischen Koadjutors Markgraf Wilhelm von Brandenburg 1529-1539, in: Gerhard Fouquet/Jan Hirschbiegel/Werner Paravicini (Hg.), Hofwirtschaft. Ein ökonomischer Blick auf Hof und Residenz in Spätmittelalter und Früher Neuzeit, Ostfildern 2008 (Residenzenforschung, Bd.21), S.77-121; Thomas Duve, Der blinde Fleck der „Oeconomia“? Wirtschaft und Soziales in der frühen Neuzeit, in: Jean-Francois KeRvéGAN/Heinz Mohnhaupt (Hg.), Wirtschaft und Wirtschaftstheorien in Rechtsgeschichte und Philosophie, Frankfurt a. M. 2004, S. 29-61.

85 S. Ellen Widder, Hofordnungen im Niedersächsischen Reichskreis, in: Höfe und Hofordnungen (wie Anm. 8) S. 457-495, hier S. 492-495. 
Jenseits all dieser wissenschaftlichen Beschäftigung mit Hofordnungen zeigt ein kurzes Zitat des Markgrafen Johann von Küstrin sehr prägnant die Bedeutung von Hofordnungen. Er begründete seine im Jahr 1561 erlassene Hofordnung mit den Worten: weil ein Unterschied sein müsse zwischen Fürstenhäusern und gemeinen Wirtshäusern ${ }^{86}$.

Zwei Blickwinkel prägten bislang die Beschäftigung mit Hofordnungen: zum einen der biographische und zum anderen der dynastische, denn man versuchte mit Hilfe der Hofordnungen die Entwicklung der zentralen Verwaltungsorgane der Territorien zu rekonstruieren. Später trat noch der kulturgeschichtliche Blickwinkel hinzu, denn Hofordnungen schienen geeignete Quellen, um den Alltag des Hofes und seine größenmäßige Entwicklung darzustellen ${ }^{87}$.

Seit dem ersten Versuch einer systematischen Erfassung und Edition von Hofordnungen durch Arthur Kern sind inzwischen über 100 Jahre vergangen, aber noch immer steht die Forschung zu Hofordnungen im Grunde an ihren Anfängen ${ }^{88}$ : Ein wirkliches Verzeichnis dieser Quellen gibt es nicht, und Editionen ${ }^{89}$ erfolgten in unsystematischer Weise und begrenzt auf jeweils nur ein Territorium.

Im Rahmen meiner Dissertation habe ich insgesamt 122 Hofordnungen aus dem Zeitraum vom 13. bis zum 19. Jahrhundert untersucht und konnte so grobe Entwicklungslinien dieser Quellengattung nachzeichnen und Besonderheiten für einzelne Territorien aufzeigen. Daraus ergab sich folgende neue, umfassend angelegte Definition für die Quellengattung Hofordnung: „Die Hofordnung ist eine schriftlich fixierte Regelung für das alltägliche Leben und das Verhalten der Menschen am Hof. Sie kann Dienstanweisungen, Zeremonialvorschriften, Organisations- und Rangklassenschemata enthalten, ebenso wie Personallisten. Ihre Bedeutung und der Umgang mit ihr zeigen an, dass sie unter die Verfassungsgesetze des Territoriums bzw. des Hofes zu rechnen ist. “90

Aus dieser Untersuchung ergab sich ebenfalls der Nachweis, dass sich die Hofordnungen in der Tat ab dem 16. Jahrhundert stark verändert haben und immer

86 Zitiert nach Max von Boenn, Die Mode. Menschen und Moden im 17. Jahrhundert, 2 Bde., München 1964, hier Bd.1, S. 236.

87 S. Ferdinand Kramer, Zur Entstehung und Entwicklung von Hofordnungen am Münchner Hof in der zweiten Hälfte des 16. Jahrhunderts, in: Höfe und Hofordnungen (wie Anm. 8) S.383-399, hier S.385-386, und HasenritTer (wie Anm.45) S.180ff. Vgl. auch Martin Hass, Die Hofordnung Kurfürst Joachims II. von Brandenburg (Historische Studien, Bd. 87), Berlin 1910, ND Vaduz 1965, S. 7.

88 Auch meine Dissertation konnte lediglich helfen, ein wenig Licht in den Dschungel der Definitionen zu bringen, s. Kircher-Kannemann (wie Anm.70).

${ }^{89}$ Kern (wie Anm.7). In den letzten Jahren erschienen folgende Editionen: FuINK/ Thissen (wie Anm.9); Wührer/Scheutz (wie Anm. 9); Kruse (wie Anm.8); Kruse (wie Anm. 61); Kruse/Paravicini (wie Anm. 8), und zuletzt Bruckhaus/Kasten (wie Anm. 9).

90 Kircher-Kannemann (wie Anm.70) S.247. Vgl. auch Ritter von Zolger (wie Anm. 50) S. 50. 
mehr Bestandteile, die man eigentlich in den Burgfriedensbestimmungen verorten würde, in sich aufnahmen.

\section{Burgfrieden und Strafen in deutschen Hofordnungen}

Wie aus den vorherigen Ausführungen deutlich wurde, veränderten sich die Hofordnungen im Verlauf des 16. Jahrhunderts und nahmen immer mehr Vorschriften bezüglich des Lebenswandels der am Hof tätigen Menschen in sich auf; Vorschriften, die zuvor allein in den Burgfriedensordnungen zu suchen gewesen waren. Offenbar versuchte man nun mit Hilfe dieser Ordnungen, soziale und zwischenmenschliche Konflikte zu entschärfen bzw. zu regeln.

In vielen Hofordnungen bilden Hinweise auf die Burgfriedensordnungen bzw. auf Strafen für Vergehen die einleitenden Abschnitte. Den Grund dafür sah Friedrich Karl von Moser in dem Umstand, dass man die Unverletzlichkeit des Regenten und seiner Familie mit Hilfe solcher Ordnungen geschützt sehen wollte. So, laut Moser, erkläre sich auch die ungemeine Strenge des Burg-Fridens ${ }^{91}$.

Offenbar erachtete man es allgemein als notwendig, solche Ordnungen zu erstellen, um einen moralischen und sittlichen Lebenswandel und vor allem ein „friedfertige[s] und gewaltfreie[s] Zusammenleben im Hofadel und Hofgesinde“ 92 zu gewährleisten. Selbst Veit Ludwig von Seckendorff, der ansonsten den Hofprediger als Dreh- und Angelpunkt der höfischen Disziplinierung ansah, war klar, dass dieser allein die Ordnung und den christlichen Lebenswandel am Hof nicht aufrechterhalten konnte. Aus diesem Grund verwies auch er auf die Hofordnungen, die neben guten Amtsträgern nötig seien, um die Ordnung des Hofes zu gewährleisten ${ }^{93}$. Auch die Verfasser der Hofordnungen selbst schienen dies so zu sehen, denn die Aufrechterhaltung der Ordnung und der Disziplin ist die mit Abstand häufigste Begründung, die für den Erlass einer neuen Hofordnung ins Feld geführt wurde ${ }^{94}$.

Um die Hofordnung allen Hofmitgliedern bekannt zu machen und sie auch im Gedächtnis zu verankern, gab es, je nach Hof und Alphabetisierungsgrad der Hofangehörigen, verschiedene Mittel: Zum einen wurde die Möglichkeit genutzt, die Hofordnungen und Burgfriedensordnungen auszuhängen, zum anderen

91 Moser (wie Anm. 4), Bd.1, S. 196.

92 Wolfgang Wüst, Luxus oder Sparzwang? Höfisches Leben im frühmodernen Kleinstaat der fränkischen Hohenzollern und der Bischöfe von Augsburg, in: Werner Paravicini (Hg.), Luxus und Integration. Materielle Hofkultur Westeuropas vom 12. bis zum 18. Jahrhundert, München 2010, S.65-82, hier S. 126.

93 Von Seckendorff (wie Anm. 39) S. 635-641.

94 Kircher-Kannemann (wie Anm. 70) S. 176-180. 
wurden sie regelmäßig verlesen oder auch jedem Hofangehörigen ausgehändigt ${ }^{95}$. Teilweise wurden diese Maßnahmen kombiniert. Insbesondere seit etwa dem 18. Jahrhundert erhielt jedes Mitglied des Hofes eine zumeist für das jeweilige Amt speziell ausgefertigte Version der Hofordnung ${ }^{96}$. Die Originale der Hofordnungen wurden durchweg von den Hofmarschall- bzw. den Hofmeisterämtern aufbewahrt.

Schaut man sich an, über welche Verfehlungen und Vergehen die Hofordnungen berichten, so sind dies vor allem Körperverletzung, Mord, Raub, Vergewaltigung und weitere schwerwiegende Delikte. Diese Angaben machen sehr deutlich, warum speziell die deutschen Höfe als „Hort des Grobianismus“ galten und man seitens des Auslandes mit zeitweilig unverhohlener Verachtung auf sie blickte ${ }^{97}$. Vor allem die ältere Kulturgeschichte führte diesen Umstand immer wieder ins Feld und so erklärt sich, gestützt auf zahlreiche Quellen insbesondere des 16. Jahrhunderts, folgende Aussage Zoepfls: „Freilich waren auch die Menschen des Spätmittelalters keine Engel gewesen. Aber was wir nun hören und sehen, das übersteigt doch die Grenzen alles bisher Dagewesenen. Johannes Agricola hat sicher recht, wenn er von seiner Zeit sagt, es lebe jetzt jedermann also roh, wild und ungezogen, daß man es nicht mehr gedenket. Es kam nun dazu, daß man sich seiner Zügellosigkeit noch rühmte, daß man mit Absicht ungehobelt war, ja daß man ein möglichst unmanierliches Benehmen für männlich und gut deutsch ansah. Grobianismus wurde Mode, Sport. Grobianus wurde nach einem Worte Sebastian Brants zum neuen Heiligen, den jedermann zu seinem Patron kürte. “98

Strafen für mögliche Delikte wurden in den Hofordnungen bereits seit den Anfängen dieser Quellengattung zumindest teilweise erwähnt und zum Teil wurden auch Strafen für verschiedene Vergehen detailliert beschrieben, so nennen etwa die 1546 und 1697 für Anhalt-Zerbst verfassten Hofordnungen den „Spanischen Mantel“ als mögliche Strafe ${ }^{99}$. In den meisten Hofordnungen aber wurde lediglich allgemein auf Strafen verwiesen, die von den jeweiligen Oberhofchargen ausge-

95 S. Wüst (wie Anm. 92) S. 126; Zimmermann (wie Anm. 29) S. 141 und Moser (wie Anm. 4), Bd.1, S. 73.

96 In $34(27,87 \%)$ der von mir untersuchten Hofordnungen wird erwähnt, dass die Ordnung regelmäßig, zumeist zwei bis viermal pro Jahr, verlesen werden sollte; in 14 (11,48 \%) Hofordnungen steht, dass sie auszuhängen und/oder an die Hofmitglieder zu verteilen sind. In 10 Ordnungen (8,20 \%) finden sich beide Angaben, s. Kircher-Kannemann (wie Anm. 70) S. 211.

97 S. Egon Friedell, Kulturgeschichte der Neuzeit. Die Krisis der europäischen Seele von der Schwarzen Pest bis zum Ersten Weltkrieg, 2 Bde., München ${ }^{9} 1991$, hier Bd. 1, S. 319.

98 F. Zoepfl, Deutsche Kulturgeschichte, 2 Bde., Freiburg 21931/1937, hier Bd. 1, S. 193.

99 Kern (wie Anm.7), Bd.2, S.23-26, und Karl Wilhelm Fürst von Anhalt, Hofordnung, Druck, Zerbst Ostern 1697, verfügbar unter http://digital.slub-dresden.de/filead$\mathrm{min} /$ data/366349732/366349732_tif/jpegs/366349732.pdf (Zugriff: 27. 08.2014). 


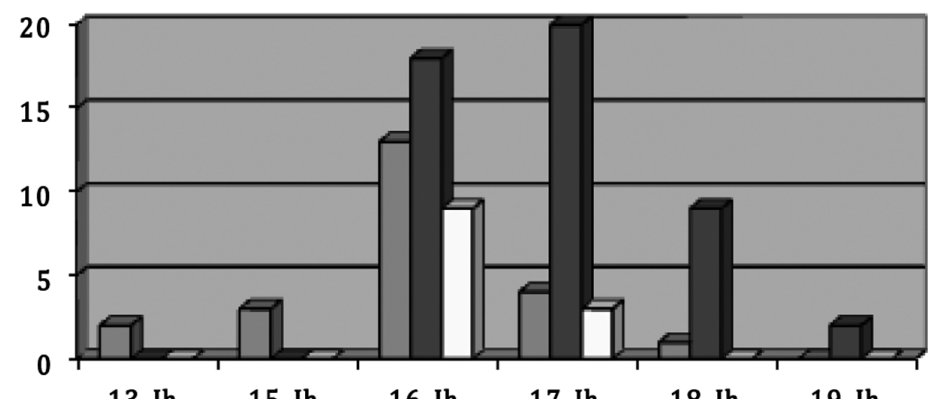

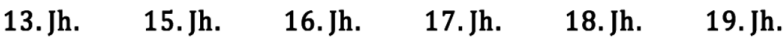

$\square_{\text {kath. }} \square_{\text {evgl. }} \square_{\text {unbek. }}$

Abb. 2: Strafen in Hofordnungen

sprochen und vollstreckt werden sollten ${ }^{100}$. Der weitaus größte Teil der Hofordnungen ${ }^{101}$ erwähnt solche hofinternen Strafen, zum Teil auch mit einem Verweis auf Vergehen, die es in der Vergangenheit gab ${ }^{102}$.

Es wird deutlich, dass die Nennung von Strafen in Hofordnungen keinesfalls eine frühneuzeitliche Erfindung ist, sondern Strafen bereits im Spätmittelalter Bestandteil dieser Ordnungen sein konnten, wenn auch in deutlich geringerem Maße als in späteren Jahrhunderten (Abb.2). Allerdings fällt auf, dass insbesondere nach dem 16. Jahrhundert und in erster Linie in protestantischen Territorien Strafen zum Inhalt von Hofordnungen gehörten.

Der Burgfrieden oder zumindest Verweise auf ihn finden sich in knapp 40 Prozent aller untersuchten Hofordnungen. Der Burgfrieden fand an protestantischen Höfen deutlich häufiger Erwähnung als an katholischen ${ }^{103}$ (Abb.3). Bislang war

100 Die häufigsten Strafen waren Entzug von Kostgeld und Essen. In gravierenderen Fällen erfolgten auch Turmstrafen oder der Verweis vom Hof, s. Kircher-Kannemann (wie Anm. 70) S.212.

10184 der untersuchten Hofordnungen behandeln im Verlauf des Textes verschiedene Strafen bei Vergehen gegen die Hofordnung oder den Burgfrieden.

10246 von 122 Hofordnungen verweisen auf Delikte, die in der Vergangenheit am Hof vorkamen, zum Teil werden sogar die Namen der Straftäter sowie die Strafen genannt, die ihnen auferlegt wurden.

103 Für den pommerschen Hof wies Hasenritter nach, dass der Burgfrieden seit dem Jahr 1560 gebräuchlich wurde und somit in direktem Zusammenhang zur Reformation stand, s. Hasenritter (wie Anm. 45) S. 153. 


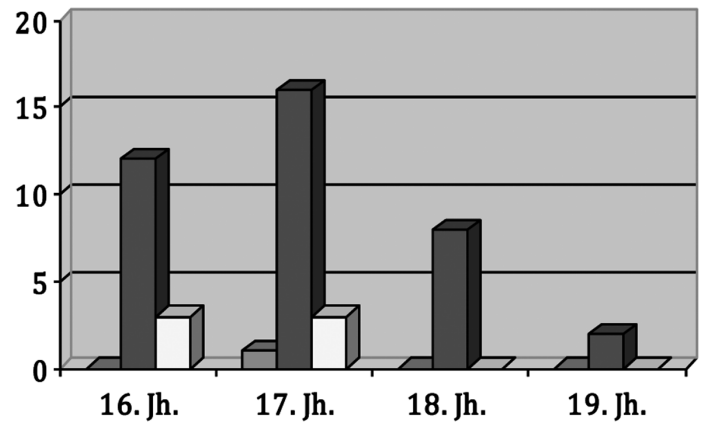

$\square$ kath. $\square$ evgl. $\square$ unbek.

Abb. 3: Burgfrieden in Hofordnungen.

nur eine einzige eindeutig katholische Hofordnung auszumachen, die den Burgfrieden als Ordnungsmaßnahme nennt. Interessant ist, dass auch im 19. Jahrhundert der Burgfrieden immer noch in Hofordnungen enthalten ist, somit also für die Angehörigen eines Hofstaats immer noch ein eigenes Recht jenseits der Landesgesetze galt ${ }^{104}$, wie vor allem in Württemberg.

Straftaten aufzuführen und gegebenenfalls sogar die Namen der Straftäter zu nennen, scheint ebenfalls ein Phänomen zu sein, das insbesondere protestantische Territorien und Hofordnungen betrifft.

In der historischen Forschung ist vielfach die Frage gestellt worden, ob die ständige Wiederholung der immer gleichen Strafen nicht darauf hindeuten könnte, dass diese Strafen im Grunde nur symbolisch waren, und man vielleicht überhaupt nicht den Versuch unternommen hat, die in den Hofordnungen und Burgfriedensbestimmungen angedrohten Maßnahmen wirklich durchzusetzen ${ }^{105}$.

104 Es gibt eine Reihe von Hofordnungen, die neben dem Burgfrieden auch auf Policeygesetze, andere Landesgesetze und auch auf kaiserliche Gesetze verweisen, so etwa die Hofordnung Herzog Ernsts des Frommen von Sachsen-Gotha aus dem Jahr 1648, die pommerschen Hofordnungen Herzog Johann Friedrichs von 1575 und Herzog Bogislaws XIV. von 1624. Gleiches gilt für die hessen-kasselsche Hofordnung aus dem Jahr 1628 von Landgraf Georg II. und nahezu alle württembergischen Hofordnungen.

${ }^{105} \mathrm{Zu}$ der Fragestellung der Bedeutung von Gesetzen in der Frühneuzeit s. Jürgen Sснцимвонм, Gesetze, die nicht durchgesetzt werden - ein Strukturmerkmal des frühneuzeitlichen Staates?, in: Geschichte und Gesellschaft (1997) S. 647-663 und Dietmar WiLLOWEIT, Gesetzgebung und Recht im Übergang vom Spätmittelalter zum frühneuzeitlichen Obrigkeitsstaat, in: Okko BeHrends/Christoph Link (Hg.), Zum römischen und neuzeit- 


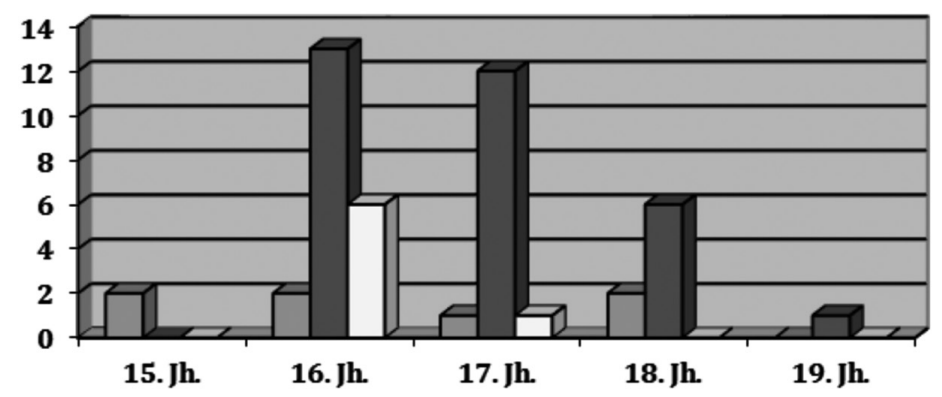

口kath. 口evgl. Dunbek.

Abb. 4: Strafen und Straftäter in Hofordnungen.

Diese Frage ist eindeutig mit „Nein“ zu beantworten: Es wurde sehr wohl versucht, Regelwerke wie Hofordnungen und auch Burgfrieden um- und durchzusetzen. Deutlich wird dies anhand zahlreicher Aktenbelege, die Auskunft geben über Anklagen wegen Verstößen gegen Hofordnung oder Burgfrieden und daraus resultierenden Verurteilungen der Delinquenten ${ }^{106}$.

lichen Gesetzesbegriff. 1. Symposium der Kommission „Die Funktion des Gesetzes in Geschichte und Gegenwart“ vom 26. und 27. April 1985, Göttingen 1987, S.123-146.

106 S. HStA Stuttgart A 44 U 1867, 1580 November 16, verfügbar unter https://www2. landesarchiv-bw.de/ofs21/olf/struktur.php?bestand=3023\&klassi=003.001.016.00036\& anzeigeKlassi $=003.001 .016 \&$ letztesLimit $=20 \&$ baumSuche $=\&$ standort $=\&$ inhaltHauptframe=lesezeichen\&setzeOlfLesezeichen=2778420 (Zugriff: 27.08.2014); HStA Stuttgart A 44 U 51751563 Mai 29 (verfügbar unter https://www2.landesarchiv-bw.de/ofs21/olf/ struktur.php? bestand=3023\&klassi=009.001.00442\&anzeigeKlassi=009.001\&letztesLimit $=440 \&$ baumSuche $=\&$ standort $=\&$ inhalt Hauptframe $=$ bestellung\&setzeBestellung $=-$ 2782383\&hinzufuegen=1 (Zugriff: 27. 08.2014); StA Wertheim G-Rep 9: Gemeinschaftliches Archiv; 3. 1501 -1600/Rezesse, Verträge und Spruchbriefe (Lade XIII-XIV) 1576 Februar 28, verfügbar unter https://www2.landesarchiv-bw.de/ofs21/olf/struktur. php? bestand $=15200 \&$ klassi $=003.00057 \&$ anzeigeKlassi $=003 \&$ letztesLimit $=40 \&$ baumSuch $\mathrm{e}=\&$ standort $=\backslash \backslash \backslash \%$ 27\&inhaltHauptframe=lesezeichen\&setzeOlfLesezeichen=1622829 (Zugriff: 27.08.2014) und HStA Stuttgart A 44 U 4495, 1560 Juli 22, verfügbar unter https:// www2.landesarchiv-bw.de/ofs21/olf/struktur.php?bestand=3023\&klassi=008.001.00307\&anzeigeKlassi=008.001\&letztesLimit=unbegrenzt\&baumSuche=\&standort $=\&$ inhaltHauptframe $=$ lesezeichen\&setzeOlfLesezeichen $=2781557$ (Zugriff: 27.08.2014). 
An allen Höfen, an denen ein Marschall anzutreffen war, oblag es diesem, die Verstöße gegen Hofordnungen und Burgfrieden festzustellen und die vorgesehenen Strafen durchzusetzen. Seine Kompetenzen veränderten sich im Laufe der Frühen Neuzeit nur wenig. Gleiches gilt für den Hofmeister an den Höfen, an denen dieser das oberste Hofamt stellte.

Sowohl von Seckendorff als auch Moser und Zedler zählten folgende Kompetenzbereiche zum Hofmarschallamt:

- die Aufsicht über den gesamten Hofstaat, inklusive der Einstellung und Vereidigung neuer Diener,

- die Aufrechterhaltung der Hofpolicey,

- die Einhaltung des Hofzeremoniells,

- die Aufwartung bei Hoffestlichkeiten und den Empfang fremder Gäste,

- die Oberaufsicht über die einzelnen Hofbereiche wie Küche, Keller und Silberkammer, das Inventar und die Mobilien des Hofhalts,

- die Organisation der Reisen und

- die Einhaltung des Burgfriedens ${ }^{107}$.

\section{Die württembergischen Hofordnungen}

Hofordnungen dienten, wie gesehen, der Organisation des alltäglichen Lebens an deutschen und anderen europäischen Höfen. Zur alltäglichen Organisation gehörten dabei aber nicht nur Anweisungen bezüglich der Mahlzeiten oder der Gottesdienstbesuche. Vor allem die allgemeine Ordnung des täglichen Lebens war es, die die Autoren dieser Anordnungen, in aller Regel die Fürsten selbst oder aber hohe Hofbeamte, gewährleisten bzw. wiederherstellen wollten.

Die allgemeine Ordnung betraf dabei vor allem den christlichen Lebenswandel, den es galt an den Höfen durchzusetzen. Daher finden sich in nahezu allen Hofordnungen des deutschen Sprachraums, insbesondere seit der Zeit der Reformation, Anweisungen zu Gottesdienstbesuchen, zu Bibellesungen, Predigten usw. Klar ist, dass der Wunsch nach einem christlichen Lebenswandel der Hofangehörigen vor allem auch die Einhaltung der Zehn Gebote umfasste, die allenthalben, wenn auch ohne direkten Verweis, beschworen wurden.

Neben den Hofordnungen, die die allgemeine Ordnung aufrechterhalten sollten und die auch durchaus Strafenkataloge für Zuwiderhandlungen beinhalteten, gab es die Burgfriedensordnungen, auf die in zahlreichen Hofordnungen als am Hof

107 S. Moser (wie Anm. 4), Bd. 2, S. 111-114; Von Seckendorff (wie Anm. 39) S. 642-658 und Art. Marschall, in: Grosses vollständiges Universal-Lexicon Aller Wissenschafften und Künste, Halle/Leipzig 1732-1750, Bd.19, S.1698-1730. Vgl. auch Alfred RITTER voN Wretschкo, Das österreichische Marschallamt im Mittelalter. Ein Beitrag zur Geschichte der Verwaltung in den Territorien des Deutschen Reiches auf urkundlicher Grundlage, Wien 1897. 
geltendes „Basisrecht“ verwiesen wird. In besonderem Maß trifft dies auf die württembergischen Hofordnungen zu, denn einzig die älteste Hofordnung Graf Ulrichs V. aus dem Jahr $1478^{108}$ verzichtet auf einen ausdrücklichen Verweis auf einen Burgfrieden und auf Strafandrohungen.

Seit dem 16. Jahrhundert, und dies entspricht den Ergebnissen der bisherigen Ausführungen, wird in den Hofordnungen der württembergischen Herzöge und späteren Könige stets auf einen Burgfrieden verwiesen. Über diesen hinaus werden weitere Strafen etwa bei Gotteslästerung oder der Störung der Tischzucht angedroht. Dies trifft erstmalig auf die recht kurze Hofordnung Herzog Christophs aus dem Jahr $1549 \mathrm{zu}^{109}$, die neben dem Burgfrieden nur die Themen der Abwesenheit des Herzogs vom Hof, der Nachtruhe, des Predigtbesuchs, der Gotteslästerung und der Tischzucht beinhaltet und alle Hofangehörigen auf die vorliegende Ordnung einschwört. Bereits ein Jahr später erließ Herzog Christoph eine weitere Ordnung ${ }^{110}$, die allerdings, anders als Kern dies sah, nicht wirklich als Hofordnung $\mathrm{zu}$ betrachten ist, sondern eher einen Zusatz zur im Vorjahr erlassenen Hofordnung darstellt. Nichtsdestoweniger werden auch hier wieder Strafen für den Fall der Zuwiderhandlung gegen die Hofordnung und die zusätzlichen Bestimmungen angedroht.

$\mathrm{Ab}$ dem 17. Jahrhundert wurden die württembergischen Hofordnungen deutlich länger und enthielten Regelungen für alle wichtigen Bereiche des alltäglichen Hoflebens. Von Herzog Johann Friedrich sind insgesamt drei Hofordnungen aus den Jahren 1611, 1614 und 1618 überliefert ${ }^{111}$, wobei sich diese drei Ordnungen bis auf geringe Abweichungen sehr ähneln und offenbar auch als Vorlage für die Hofordnung Markgraf Philipps von Baden-Baden dienten ${ }^{112}$. Einleitend wird in diesen Hofordnungen darauf verwiesen, dass sie dazu dienen sollen, die Disziplin der Hofbediensteten zu erhalten. Alle hatten einen Eid auf diese Hofordnung zu leisten, die insgesamt zweimal pro Jahr verlesen werden sollte und über deren Einhaltung der Haushofmeister sowie der Burgvogt zu wachen hatten. Auf diese Einleitung folgt bereits die erste Strafandrohung, die jeden treffen sollte, der sich nicht nach den christlichen Grundsätzen richtete oder etwa zu früh die Predigt verließ. Erst jetzt folgt eine Einschwörung auf den Fürsten selbst, von dem Schaden abzuwenden sei. Hier waren es allerdings nicht Haushofmeister und Burgvogt, die zu Strafen berechtigt waren, sondern der Haushofmeister, der Landhofmeister und fast selbstverständlich - der Herzog selbst.

108 Ediert bei Hofacker (wie Anm. 7) S. $224 \mathrm{ff}$.

109 Ediert bei Kern (wie Anm.7), Bd.2, S.141 f.

110 Ediert ebd., Bd.2, S. $142 \mathrm{f}$.

111 Ediert ebd., Bd.2, S.143-161. Vollständig ediert ist hier die Ordnung aus dem Jahr 1614. Die anderslautenden Teile der Ordnungen der Jahre 1611 und 1618 sind in den Fußnoten vermerkt.

112 S. ebd., Bd.2, S. $114-124$. 
Der vierte und ausgesprochen lange Abschnitt ist dem Burgfridt gewidmet, der mit wortten, werckhen und geberden vestiglich gehallten solle werden. Wer aber den andern mit wortten schmehen oder hochmuetten, hinaußfordern, trauen, fluechen, vil weniger schlagen, stechen, stoßen oder sonsten in einichen weeg frevenlich beleidigen solle, der solle die nun aufgeführten Strafen erfahren ${ }^{113}$. In der Edition zieht sich dieser Strafenkatalog über nicht weniger als drei Seiten. Die niedrigsten Strafen sind Turmstrafen bei Wasser und Brot, die höchsten Strafen sind Todesstrafen und es wird deutlich, dass das Strafmaß abhängig war vom Stand und der Position des Delinquenten:

Begebe es sich dann, daß unser ober[-] oder Underofficierer mit der Wehr, Dolchen, Meßer, Spieß oder andern Waffen thätlich von jemandt angefallen würden, solche Thäter sollen am Leben gestrafft werden. Beschehe es aber under dem gesindt, solle der frevler die rechte Handt velobren haben ${ }^{114}$.

$\mathrm{Zu}$ den Burgfriedensbestimmungen gehörte es auch, den Hofangehörigen einzuschärfen, dass sie sich nicht nur bei Hof und im Burgfriedensbezirk tadellos zu verhalten hatten, sondern auch an allen anderen Orten, an denen sie sich aufhielten. Dies macht deutlich, dass spätestens seit Beginn des 17. Jahrhunderts der Hof und somit auch die Hofangehörigen als „Aushängeschild“ des Fürsten angesehen wurden, die dazu dienten, seine Reputation zu heben und ihn auch nach außen zu repräsentieren.

Diesen Aspekt hatte auch fast zur gleichen Zeit Georg Engelhart Löhneysen in seiner „Aulico Politica“ als besonders relevant hervorgehoben. Er verfasste zwei als beispielhaft gedachte Hofordnungen und setzte ihnen folgende Aussage voran: ist nachfolgente Ordnung gleichermassen darauff dirigiret onnd angestellet / wie sich ein jeder in dem onnd sonsten fleissig und trewlich erzeigen / und seines Ampts und Beruffs warten sol / damit des Fürsten reputation und ansehen erbalten und nicht geschmälert werde ${ }^{115}$.

Weitere Themen, die nach den Burgfriedensbestimmungen in den Hofordnungen Herzog Johann Friedrichs behandelt werden, sind die Mahlzeiten, zu welchen Uhrzeiten sie stattfinden sollen, und wie man sich bei ihnen zu verhalten habe, sowie der allgemeine Lebenswandel, die Abschaffung der Ausspeisung, außer im Krankheitsfall, der vom Hofarzt attestiert werden muss, die Anzahl der Knechte, die den Hofadeligen zur Verfügung stehen, der Zustand der Rüstungen, das Verhalten bei Jagden, das Abreiten, also das Verlassen des Hofes, die Verteilung des Pferdefutters, der Pferdekauf, die Verteilung der Lichter durch den Lichtkämmerer, das Verhalten in Notfällen und bei Gefahren, das Schießen, das nur im Feld erlaubt ist, die Versorgung der Hunde und das Herbergsgeld. Beendet werden diese drei Hofordnungen mit dem Hinweis an die Ritterschaft, dass sie auf ihr

\footnotetext{
113 Ebd., Bd.2, S. 146.

114 Ebd., Bd.2, S. 147.

115 Löhneysen (wie Anm. 5) S. 350.
} 
Gesinde und dessen Verhalten zu achten habe und dass alle Hofangehörigen sowohl die Hofordnung als auch die Befehle des Haushofmeisters und Burgvogts befolgen sollen.

Nach diesen drei sehr ausführlichen Hofordnungen klafft, zumindest was die bekannten und edierten Hofordnungen Württembergs angeht, eine Lücke von fast 100 Jahren. Die nächste Hofordnung stammt von Herzog Eberhard Ludwig aus dem Jahr 1711 und wurde bereits von Friedrich Karl von Moser in seinem „Teutschen Hof-Recht" ediert ${ }^{116}$. Auch sie ist wieder ausgesprochen lang und ausführlich und baut inhaltlich eindeutig auf den Hofordnungen des frühen 17. Jahrhunderts auf. Über mehr als zwei Seiten erstreckt sich in der Edition der einleitende Teil, der eine Einschwörung auf einen christlichen Lebenswandel und die Hofordnung zum Inhalt hat. Anders als in den vorangegangenen Hofordnungen sind nun aber nicht mehr der Haushofmeister und der Burgvogt die obersten Hofbeamten, die über die Einhaltung aller Ordnungen zu wachen haben. Ein neues Amt hat sich an die Spitze des Hofhalts gesetzt: das Marschallamt ${ }^{117}$. Oberhofmarschall und Marschall obliegt nun die Aufsicht. Ausdrücklich werden aber auch alle anderen Oberhofvorgesetzten in die Pflicht genommen, die Einhaltung der Hofordnung und des Burgfriedens zu überwachen ${ }^{118}$.

Erneut ist es dann die Predigt und der Besuch der Gottesdienste, die einen breiten Raum in der Hofordnung einnehmen ${ }^{119}$. Die gestiegene Bedeutung zeremonieller Aspekte äußert sich deutlich in der Anweisung, dass sowohl die Kavaliere als auch das übrige Gesinde des Hofes vor der Predigt dem Fürsten an seinen Gemächern aufwarten sollen. Der dritte Punkt der Hofordnung betrifft wiederum den Fürsten, wie schon 100 Jahre zuvor: Alle Hofangehörigen sind dazu verpflichtet, Schaden von ihm abzuwenden und im Falle, dass sie etwas Verdächtiges hören, müssen sie sofort Meldung an den Oberhofmarschall, den Hofmarschall, die Hofratsstube, die geheimen Räte oder den Fürsten selbst machen ${ }^{120}$.

Als vierter Punkt, folgt - auch dies ist gleich geblieben - der Burgfrieden ${ }^{121}$, und wieder sind es vor allem Delikte wie Fluchen, Trinken, Diebstahl und Tätlichkeiten, die unter Strafe gestellt werden; dabei wird deutlich unterschieden, ob dieses Delikt von einem Adeligen oder einem einfachen Hofbediensteten ausgeübt wurde und gegen wen es verübt wurde, ob gegen einen Oberhofvorgesetzten, einen Bedienten einer fremden Herrschaft oder gegenüber einem Gleichgestellten. Erstaunlich ist, dass sogar die Sitz- und Tischordnung bei Hof offenbar unter den Burgfrieden fällt, und ein Verstoß gegen sie entsprechend harte Strafen nach sich zieht.

\footnotetext{
116 Moser (wie Anm.4), Bd.1, Anhang, S. 54-73.

117 Zur Bedeutung der Namensänderung des obersten Hofamtes s. Kircher-KanneMANN (wie Anm. 70) S. 183-188.

118 Moser (wie Anm. 4), Bd.1, Anhang, S. $54 \mathrm{ff}$.

119 Ebd., Bd.1, Anhang, S. 56 f.

120 Ebd., Bd.1, Anhang, S. 57.

121 Ebd., Bd. 1, Anhang, S. $57 \mathrm{ff}$.
} 
Insbesondere das Abschleppen - also das Entwenden von Silber- und Zinngeschirr, sowie von Porzellan und Tischwäsche - wird ausführlich angesprochen; ein Delikt, das offensichtlich an allen deutschen Höfen ein massives Problem darstellte und oftmals breiten Raum in den Hofordnungen einnimmt ${ }^{122}$. Offenbar war das Problem am württembergischen Hof so groß, dass man diesen Passus nun in den Burgfrieden integriert hat. In den Jahren zuvor wurde es noch im nachfolgenden Abschnitt über die Mahlzeiten behandelt ${ }^{123}$. Dieser Abschnitt folgt auch wieder auf den Burgfrieden, ist nun aber deutlich stärker zeremoniell und organisatorisch ausgerichtet. Nahezu gleich geblieben ist auch der darauffolgende Abschnitt über den Lebenswandel der Hofangehörigen ${ }^{124}$.

Neu ist die Regelung bezüglich des Zutritts zu Küche und Keller sowie die über die Zulassung zum Essen, insbesondere der Adeligen, die nun die Erlaubnis des Oberhofmarschalls oder Hofmarschalls benötigen ${ }^{125}$. Ebenfalls neu ist die Regelung des Abtritts, der nur noch an den dafür vorgesehenen Stellen stattfinden darf, worüber die Wächter die Aufsicht haben ${ }^{126}$. Auch neu ist der Abschnitt über das Verhalten der Hofangehörigen in der Stadt und die Anweisung, dass dort sowohl der Vogt als auch der Bürgermeister darauf sehen sollen, dass die Hofbediensteten weder Alkohol trinken noch spielen oder lärmen ${ }^{127}$.

Die letzten Kapitel der Hofordnung behandeln die Themen: Abreiten und Kostgeld, Heimleuchten, Knechte und Diener, die gute Zeugnisse vorweisen müssen, um am Hof arbeiten zu dürfen, wer die Erlaubnis erhält mit dem Fürsten zu reisen, das Schießen, das einer speziellen Erlaubnis bedarf, die Verteilung der Lichter und Nachttöpfe ${ }^{128}$, das Verhalten bei Notfällen, nochmals ein Verbot des Schießens, worauf der Vogt zu achten habe, das Verbot von Hunden und das Herbergsgeld. Der letzte Abschnitt gleicht den vorangegangenen Hofordnungen und beinhaltet das Gebot, die Hofordnung einzuhalten und den Befehlen der Vorgesetzten, insbesondere des Oberhofmarschalls und Hofmarschalls, Folge zu leisten ${ }^{129}$.

Es fällt auf, dass alle militärischen Aspekte aus der Hofordnung verschwunden sind. Offenbar hat in den 100 Jahren zwischen 1611 und 1711 eine enorme Wandlung der höfischen Welt stattgefunden. Finden wir in den Ordnungen aus den Jahren 1611 bis 1618 noch Anweisungen bezüglich Rüstungen, Pferden, Bewaffnung, Pferdekauf und Verhalten beim Feldreiten und auf Jagden, so sind diese 1711 voll-

122 S. Kircher-Kannemann (wie Anm. 70) S. $214 \mathrm{f}$.

123 Moser (wie Anm.4), Bd.1, Anhang, S. 63-66.

124 Ebd., Bd.1, Anhang, S. 66.

125 Ebd., Bd.1, Anhang, S.66f.

126 Ebd., Bd.1, Anhang, S.67f.

127 Ebd., Bd.1, Anhang, S. 68.

128 Auch die Nachttöpfe sind neu und machen deutlich, dass man offenbar versuchte, vor allem die Sauberkeit des Hofes zu verbessern, um so eine positivere Außenwirkung zu erzeugen.

129 Moser (wie Anm.4), Bd.1, Anhang, S. 72 f. 
kommen verschwunden. Der Hof ist ein repräsentativer, ein zeremonieller Hof geworden; Wehrhaftigkeit und militärisches Leben sind gewichen.

Gut 80 Jahre später erscheint die Hofordnung erneut wie ausgewechselt: der Ton wird „amtlich“, und die Hofordnung Herzog Ludwig Eugens ${ }^{130}$, die nun den Titel Ordnung und Sazungen trägt, erhält eine völlig neue Einleitung:

Da Wir in Verwaltung Unsers Regenten-Amts vorzüglich auch Unser Augenmerk dabin richten, daß alle Stände Unsers Herzogthums mit guten Ordnungen verseben, die ältere theils aufrecht erhalten und eingeschärft theils auch wieder erneuert und besonders in Hofsachen nach Beschaffenheit der Umstände und Zeiten eingerichtet werden; Als haben Wir auch keinen Anstand gefunden, die von Unseres Höchstseligen Herrn Bruders Liebden in anno 1779. Neu revidirte Hof-Ordnung nach einig wenigen Abänderungen zu bestättigen. In dieser Rucksicht machen Wir hierdurch einen Unterschied unter Unsern Hof-Officianten, auch Garderobe-und samtlich niedern Dienerschaft; wollen jene Beobachtung des Ihnen bei dem Antritt ibres Amts zustellenden besonders Staats, so gnädigst als ernstlichst verwiesen haben. Bei der andern Classe hingegen befehlen Wir hiemit, daß die darunter begriffene Personen folgende Ordnungen und Satzungen sowol in denen Residenzen zu Stuttgart und Ludwigsburg, als auch auf dem land vest und unverbrüchlich balten sollen $[\ldots]^{131}$.

Erstmals also wird hier auf eine ältere Ordnung verwiesen ${ }^{132}$ und eine gesonderte Begründung für die Erstellung der neuen Hofordnung angeführt. Es ist nun auch nicht mehr der christliche Lebenswandel, der zuerst beschworen wird, sondern die Treue und der Gehorsam gegenüber dem Fürsten und seinen Beamten. Auch der Fleiß wird nun ausdrücklich erwähnt, der von allen Hofangehörigen gefordert wird. Altbekannt hingegen ist die Wendung, dass wo jemand dieser Unserer Hofdiener etwas an Worten oder Werken hören, vernebmen, oder vermerken würde, so Unserer Höchsten Person oder Unserem Herzoglichen Hause zum Schaden oder Nachtheil gereichen könnte ${ }^{133}$, er dies umgehend zu melden hat. Allerdings bleibt diese Meldepflicht nun nicht mehr auf den Regenten und seine nähere Umgebung beschränkt, sondern umfasst auch Unsere Herzogliche Ministers, Hof-Militair-Canzley und überhaupt alle Unsere getrene und liebe Unterthanen ${ }^{134}$.

Erst jetzt folgt ein Abschnitt über den christlichen Lebenswandel, dessen sich alle zu befleißigen haben und den regelmäßigen Besuch der Gottesdienste. Der dritte Abschnitt beinhaltet, wie dies schon in der Hofordnung des Jahres 1611 war, den Burgfrieden; allerdings wird dieser nun nicht mehr in die Hofordnung ein-

${ }^{130}$ Hofordnung Herzog Ludwig Eugen von Württemberg-Teck (wie Anm. 7).

131 Ebd., S.3f.

132 Leider liegt mir die Hofordnung von 1779 nicht vor.

133 Hofordnung Herzog Ludwig Eugen von Württemberg-Teck (wie Anm. 7) S. 5.

134 Ebd. 
gearbeitet, sondern ist nur noch als Verweis aufgenommen, ebenso wie ein eigens ergangenes Duell-Edict. Bezüglich des Burgfriedens heißt es:

Ordnen und befeblen Wir, daß nicht nur in den Residenzen, sondern auch an all andern Orten, wo Wir Uns entweder mit dem gewöhnlichen, oder auch nach Unserem Woblgefallen mehr eingeschränkten Hof-Staat befinden, ein aufrecht und unverbrüchlicher Burgfried, mit Worten, Werken und Gebärden vestiglich gehalten, und derjenige, so darwider handelt, mit der empfindlichsten Strafe angeseben werden solle. Wie Wir dann zu desto besserer Beobachtung dieser Unserer Verordnung das unterm 6. Juny 1738 ergangene Duell-Edict seinem ganzen Innhalt nach, wo Wir nicht in nachstehenden Puncten ein anders verordnen, hieher wiederholen $^{135}$. Der Burgfriedensbezirk wird auch in dieser Hofordnung erneut detailliert beschrieben, damit sich der Unwissenheit dißfalls niemand entschuldigen könne ${ }^{136}$.

Punkt fünf der Hofordnung thematisiert das Verhalten gegenüber Vorgesetzten und verweist auf eine Criminal-Strafe bei Zuwiderhandlung ${ }^{137}$. Im Falle, dass jemand streitet, flucht, trinkt oder schmäht, so bekommt er zunächst eine milde Strafe und erst im Wiederholungsfall droht die Hofordnung mit Leibstrafen oder Kassation, sprich Dienstenthebung. Erneut wird dann der Gehorsam thematisiert, der seitens der Hofbediensteten unbedingt den Vorgesetzten entgegengebracht werden muss, denn da die Subordination bei allen Ständen die Seele der Ordnung ausmachet; so wollen Wir auch Unserer niedern Hofdienerschaft die strengste Beobachtung des schuldigen Respects und Gehorsams gegen die Vorgesezte nachdrücklichst befoblen haben ${ }^{138}$.

Neu ist Punkt acht, der darauf verweist, dass man sich gegenüber den Dienern von Gästen korrekt zu verhalten habe. Das Tragen von Waffen und das Schießen (Punkt neun) hingegen waren auch schon in früheren Hofordnungen enthalten.

Bereits die Hofordnung des Jahres 1711 war intensiv auf die Problematik der Diebstähle bei Hof eingegangen, und so verwundert es nur wenig, dass dieses Thema auch im Jahr 1794 noch einen breiten Raum in der Hofordnung einnimmt. Neu allerdings ist, dass hier nun auf ein General-Rescript verwiesen wird, das am 16. Oktober 1718 ergangen ist - also nur sieben Jahre nach der zuletzt angeführten Hofordnung -, das weiterhin Gültigkeit besitzt und wonach in Fällen des Hofdiebstahls die Täter bestraft werden sollen ${ }^{139}$. Trotz dieses Verweises werden unter den Punkten 12 und 13 nochmals insbesondere die Diebstähle von Porzellan und Silber und das Abtragen als Delikte genannt, die mit Kassationsstrafe belegt sind.

Dass der Zutritt zu Küche und Keller geregelt wird, hatten wir auch bereits in der Hofordnung von 1711 gesehen, nun tritt hierzu noch eine Regelung über den

\footnotetext{
135 Ebd., S. 6 .

136 Ebd.

137 Ebd., S. 7 f.

138 Ebd., S. 8.

139 Ebd., S. 10 f.
} 
Zutritt zur Apotheke ${ }^{140}$. Dies könnte darauf hindeuten, dass diese entweder neu war oder aber es in der Zwischenzeit dort größere Probleme gegeben hat.

Als eigenständiger neuer Punkt findet sich nun auch die Aufsicht über die niederen Tische, bei denen die Hoffouriere besonders darauf zu achten haben, dass es dort ordentlich und anständig zugeht. Punkt 16 betont, dass wer aufwartet reinlich sein und eine ordentliche Livrée tragen soll ${ }^{141}$.

Ein Problem, das in dieser Hofordnung erstmals zutage tritt, ist das des Einforderns von Geschenken. Offenbar hatten es sich die Hofbediensteten angewöhnt, von hochrangigen Gästen Geschenke einzufordern, und das offenbar auf vehemente Art. Aus diesem Grund war bereits am 6. November 1755 eine Verordnung ergangen, auf die hier in Punkt 17 verwiesen wird ${ }^{142}$. Die genannte Strafe ist auch hier wieder die Kassation.

Die Regelung des Zutritts zu den fürstlichen Räumen ist ebenfalls neu und wird vor allem mit dem Problem des Geheimnisverrats begründet:

Diejenige Unserer Officianten und Hofbedienten, welche in die Herzogliche Zimmer-oder Arbeits-Cabinet, diensthalber den Eingang haben, sollen sich nicht erkühnen, wenn Papire allda befindlich, in solchen zu blättern, und deren Innhalt zu erforschen; überhaupt aber Unserer ganzen Hofdienerschaft nachdrücklichst untersagt seyn, von demjenigen, was sie etwa bei Aufwartungen oder sonst, von Regierungs- Militair-oder andern Sachen sehen oder hören werden, jemand etwas davon zu offenbaren; wie dann alles Raisoniren über Staats-Regierungs- und andere dergleichen Sachen Unserer Hofdienerschaft sowohl unter sich als mit Fremden und bedienten der Gesandten oder anderer Herrschaften, obnehin bei schwerer unausbleiblicher Strafe verbotten ist ${ }^{143}$.

Die beiden nachfolgenden Punkte, die das Verhalten in Notfällen, insbesondere bei Feuer und auf Reisen betreffen, waren bereits in der zuvor beschriebenen Hofordnung enthalten.

Anders sieht dies mit Punkt 21 aus, in dem die Ausgabe der Livréen an die Hofdienerschaft geregelt wird: So sollen sie alle vier Jahre eine neue Sonntags-Livrée und alle zwei Jahre eine neue Alltags-Livrée erhalten, die sie unter Androhung von Strafen pfleglich behandeln und verwahren sollen ${ }^{144}$.

Urlaub ist ein neues Wort in württembergischen Hofordnungen. Sprachen die vorangegangenen Ordnungen etwa von Abreiten, wenn es um das Verlassen des Hofes ging, so wird nun Urlaub zugestanden, wobei dessen Länge genau einzuhalten ist ${ }^{145}$. Ebenfalls neu ist der Verweis auf die Policeyordnung und die Unterstellung der gesamten Hofdienerschaft unter eben diese Verordnungen:

140 Ebd., S. 13.

141 Ebd., S. $13 \mathrm{f}$.

142 Ebd., S. 14.

143 Ebd., S. 15.

144 Ebd., S. 16.

145 Ebd., S. $16 \mathrm{f}$. 
Allen Verordnungen, welche in Policey-Sachen als wegen Beberbergung fremder Personen, Gassensäuberung-Feuer-oder Bettel-Anstalten und dergleichen, von Unserer Herzoglichen Policey-Deputation oder sonst ergehen, ist die gesamte Hofdienerschaft ebenfalls ganz unterworfen, und hat sich bei denen in diesen Gesezen bestimmten Strafen nach solchen Befeblen zu achten ${ }^{146}$.

Dass am Ende der Hofordnung nochmals ausdrücklich darauf hingewiesen wird, dass diese auch einzuhalten sei, ist ein Brauch, der uns schon bei den ersten Hofordnungen begegnete und der sich auch hier wiederholt. Allerdings gibt es auch eine Neuerung, und das ist das Prinzip von Zuckerbrot und Peitsche, denn so wie allen Übertretern dieser Ordnung Strafe angedroht wird, so werden nun alle, die die Ordnung einhalten, auch Unserer höchsten Gnade und huldreichen Versorgung versichert ${ }^{147}$.

Ebenfalls neu ist Punkt 25, der darüber informiert, dass Damit sich endlich Niemand mit der Unwissenheit dieses Gesezes entschuldigen kann, der Herzog solches durch den Druck allgemein bekannt machen lässt und befiehlt, dass einem jeden Unserer Herzoglichen Officianten und Hofdiener ein Exemplar davon zugestellt, auch daß diese Hof-Ordnung alle Jahr einmal, wann der Hof in Stuttgart beisammen, in Anwesenheit eines Maitre von Hof, den samtlichen Officianten und ganzen niedern Dienerschaft sowobl von Unserer Herzoglichen Garderobbe, als andern Hof-Bedienten, ingleichem denen in Pflicht und Besoldung, folglich unter der Hof-Jursidiction stebenden Hof-Handwerksleuten, und überbaupt allen denenjenigen Hofdienern, welche hierunter begriffen, auch denen in das Herzogliche Schloß Dienstwegen kommenden Cavaliers-Bedienten, vorgelesen werden solle ${ }^{148}$.

Herzog Friedrich II. erließ kurz nach dem Antritt seiner Regentschaft eine neue Hofordnung. Diese datiert vom 20. April $1798^{149}$ und ist bis auf wenige Wendungen identisch mit der Hofordnung des Jahres 1794, die, wie wir ja sahen, auf einer Hofordnung des Jahres 1779 beruhte, so dass also über zirka 20 Jahre hinweg letztlich ein und dieselbe Ordnung mit nur ausgesprochen geringen Abwandlungen in Kraft blieb. Eine Modifikation in der Formulierung der Hofordnung tritt erst wieder mit einer einschneidenden historischen Veränderung auf und zwar mit der Annahme der Königswürde durch Friedrich II. am 1. Januar 1806.

Gut ein Jahr später, am 22. März 1807 wurde eine neue Hofordnung erlassen, denn Wir Friderich, von Gottes Gnaden, König von Württemberg etc. [...] finden Uns bewogen, die unterm 20. April Anno 1798 revidirte Hof-Ordnung allergnädigst [...] theils zu bestätigen, theils zu erneueren ${ }^{150}$. Diese Hofordnung ist in mehrfacher Hinsicht neu, denn nicht nur, dass ihre Aufteilung in fast keinem Punkt mehr einer der Vorgängerordnungen entspricht, sie ist auch als erste Hofordnung

\footnotetext{
146 Ebd., S. 17.

147 Ebd.

148 Ebd., S. 18.

149 Hofordnung Herzog Friedrich II. von Württemberg-Teck (wie Anm. 7).

150 Hofordnung König Friedrich (wie Anm. 7) S. 87-93, hier S. 87.
} 
Württembergs einige Jahre später, im Jahr 1839, vollständig in einer württembergischen Gesetzessammlung veröffentlicht worden. Eigentlich ein Novum, denn zuvor galten Hofordnungen in den meisten Territorien als "Staatsgeheimnisse“, und es war sogar zum Teil unter Strafe gestellt, ihren Inhalt gegenüber Außenstehenden preiszugeben ${ }^{151}$. Ein Phänomen mit dem auch Friedrich Karl von Moser zu kämpfen hatte, als er für sein „Teutsches Hof-Recht“ auf der Suche nach Hofordnungen war, die er edieren konnte ${ }^{152}$. Auch Johann Christian Lünig musste sich für sein „Theatrum Ceremoniale“ auf ausgesprochen alte Hofordnungen zurückziehen, einzig neueren Datums in seiner Edition sind Hofstaatslisten ${ }^{153}$. Allerdings hatte schon die Edition der 1711 verfassten Ordnung durch Moser gezeigt, dass man in Württemberg hier durchaus andere Wege ging.

Der Anfang der Hofordnung von 1807 ähnelt noch der vorangegangenen, denn zunächst werden wieder alle Hof-Officianten auf ihre eidlich beschworenen Pflichten verwiesen und darauf, dass sie ihren Dienst treu, fleißig und gehorsam zu versehen haben. Ebenfalls gleich blieb der Hinweis darauf, dass jeder Schaden vom königlichen Haus abzuwenden sei und sich alle Hofangehörigen eines christlichen Lebenswandels zu befleißigen hätten. Vor allem die Trunksucht sei es, der zu widerstehen sei und die mit Arrest oder gar Entlassung bestraft werde ${ }^{154}$. Auch liederliches Verhalten wird angeprangert und das Saufen und Lärmen in Wirtshäusern, ebenso auch das Beschmutzen der Livrée und das Ausgehen ohne dieselbe. Es wird also vor allem stark darauf geachtet, dass die Außenwirkung, die Repräsentation des gesamten Hofstaates stets den vom König gesetzten Maßstäben entspricht; so entsteht eine Disziplinierung, die bis hinein in die Kleidung reicht.

Und selbst jetzt, zu Beginn des 19. Jahrhunderts, wird noch auf den Burgfrieden verwiesen, der vor allem an den Orten einzuhalten sei, an denen sich der König selbst befindet. Wiederum wird auch der Burgfriedensbezirk genau beschrieben. Der einleitende Satz von Punkt sechs ist ebenfalls aus den Vorgängerordnungen übernommen: Da die Subordination bei allen Ständen die Seele der Ordnung ausmachet ${ }^{155}$. Hier geht es erneut darum, den Befehlen der Vorgesetzten Folge zu leisten, und als Strafen bei Nichtbefolgung finden sich wiederum Kassation, sowie Leibes-Zucht-oder Vestungs-Strafe $[\mathrm{n}]^{156}$. Punkt sieben der Ordnung befasst sich mit dem allgemeinen Benehmen der Bediensteten, und Punkt acht verbietet das Schießen sowohl in der Stadt als auch auf dem Feld. Auch die Diebstähle scheinen nicht wirklich nachgelassen zu haben, denn ihnen wird mit Punkt neun ein aus-

151 S. etwa die Hofordnung Markgraf Johanns von Küstrin aus dem Jahr 1561, ediert bei Kern (wie Anm.7), Bd.1, S.34-82.

152 Moser (wie Anm. 4), Bd. 1, Vorbericht.

153 Johann Christian LüNIG, Theatrum Ceremoniale Historico-Politicum, 3 Bde., Leipzig 1719-1721, hier Bd.2, S. 1473-1523.

${ }_{154}$ Hofordnung König Friedrich (wie Anm. 7) S. 88.

155 Ebd., S. $88 \mathrm{f}$.

156 Ebd., S. 89. 
gesprochen ausführlicher Absatz gewidmet, mit Verweis auf die peinliche Halsgerichts-Ordnung. Auf spezielle Diebstähle, etwa von Porzellan und Silber, gehen dann Punkt zehn und elf der Hofordnung ein ${ }^{157}$.

Waren es in der vorherigen Hofordnung der Zutritt zu Küche, Keller und Apotheke, die geregelt bzw. verboten wurden, so kommen nun entsprechende Regelungen auch für die Kaffeekammer, die Backkammer und die Konditorei hinzu. Die nachfolgenden Abschnitte 14 bis 24 entsprechen den Abschnitten 15 bis 25 der Hofordnung von 1794 bzw. 1798 mit nur geringfügigen Änderungen, wie etwa, dass die Livréen nun jährlich bzw. dreijährlich erneuert werden.

Eine nochmalige Umstrukturierung erfuhr die württembergische Hofordnung im Jahr $1818^{158}$. In den Jahren zwischen 1807 und 1818 war die gesamte Hofverwaltung umstrukturiert worden, und eben jene Umstrukturierung findet sich in den Ausführungen der neuen Hofordnung wieder ${ }^{159}$. Diese neue Hofordnung wurde bereits im Jahr ihres Erscheinens im „Königlich Württembergischen Staatsund Regierungsblatt" abgedruckt und beginnt wieder mit den allgemeinen Dienstpflichte[n] der Hof-Officianten und Diener. Neu ist, dass den Hofbedienten nun die Möglichkeit eingeräumt wird, Einsprüche gegen die Anordnungen ihrer Vorgesetzten einzureichen, und ein Instanzenweg vorgegeben wird ${ }^{160}$.

Die darauffolgenden Punkte sind zwar in anderer Reihenfolge geordnet als bisher, aber inhaltlich den älteren Ordnungen vergleichbar: So geht es um die Ermahnung, den Dienst ordentlich zu versehen, das Verhalten bei Bränden und die Anzeige von Dienstvergehen. Neu hinzu gekommen ist $\$ 6$ mit dem Titel Art des Verfahrens bei Entlassung. Verwiesen wird hier auf eine am 20. Dezember 1816 erlassene Verordnung, die $1817 \mathrm{im}$ Staats- und Regierungsblatt veröffentlicht worden ist.

Auch $\ 7$ gehört zu den Neuerungen dieser Hofordnung: Erstmals wird den Hofbediensteten ein ordentliches Ruhegehalt in Aussicht gestellt. Voraussetzung für den Erhalt desselben ist, dass sie wenigstens 10 Jahre vorwurfsfrei gedient haben. Eine solche Regelung gab es bis zu diesem Zeitpunkt für Hofbedienstete nicht, sie waren abhängig vom Wohlwollen des Regenten, hatten aber keinerlei rechtlich begründeten Ansprüche ${ }^{161}$. Auch die Paragraphen acht bis zehn stellen Neuerungen dar und sorgen für eine größere Rechtssicherheit der Hofbediensteten, denn erneut werden Beschwerdestellen genannt, und es erfolgt eine genaue Regelung, wer unter die Hofgerichtsbarkeit oder aber unter die staatliche Gerichtsbarkeit fällt.

157 Ebd., S. 90 f.

158 Königlich-Württembergisches Staats- und Regierungs-Blatt vom Jahr 1818, Stuttgart 1818, S. 345-353.

159 Vgl. Eberhard FriTz, Knecht, Kutscher, Koch, Kammerdiener, König. Zur Sozialgeschichte des königlichen Hofes in Württemberg (1806 bis 1918), in: ZWLG 66 (2007) S. 249-292, hier S.263.

160 Württembergisches Staats- und Regierungs-Blatt (wie Anm. 162) S. 345.

161 Ebd., S. 346 und vgl. Fritz (wie Anm. 159) S. 263. 
Die Unterordnung unter die lokalen Polizeistellen war bereits in der Hofordnung von 1807 thematisiert worden und stellt insofern keinen neuen Punkt dar. Anders sieht es aus mit $\int$ 13: Handhabung der Polizei im Innern der Hofgebäude ${ }^{162}$. Sie obliegt nun nicht mehr dem Hofmarschall, sondern dem Hausverwalter, der in dieser Beziebung dem Ober-Hofbeamten [...] sonst aber der Königl. Schloßhauptmannschaft unmittelbar untergeordnet ist ${ }^{163}$. Wie schon in den vorangegangenen Hofordnungen gesehen, stellte der Hofdiebstahl offenbar ein großes Problem dar und an diesem hatte sich auch im Jahr 1818 nichts geändert, wie wir den Paragraphen 15 bis 18 entnehmen können.

Der Burgfrieden sowie der Burgfriedensbezirk wurden nicht, wie man vielleicht vermuten könnte, aus der Hofordnung gestrichen - im Gegenteil: Die Bestimmungen bezüglich des Burgfriedens nehmen nun sogar wieder einen breiteren Raum ein und werden in den Paragraphen 19 bis 27 behandelt und zwar je nach Grad und Schwere des jeweiligen Verstoßes gegen den Burgfrieden ${ }^{164}$.

Die bereits bekannte Bestrafung der Geschenkannahme findet sich in $\$ 29$ wieder und auch die jährliche Publikation der Hofordnung sowie das Verlesen sind noch immer Bestandteil der Hofordnung, hier hat sich seit 1794 nichts verändert. Was sich allerdings verändert hat, ist, dass nun alljährlich die Publikation zum Anlass genommen werden soll die Officianten sämmtlicher Stäbe über das Benebmen eines jeden ibnen untergebenen Dieners im Allgemeinen, so wie über seine Qualifcation zum Dienste zu vernebmen ${ }^{165}$. Hierüber sind Notizen zu machen und diese werden dann vom Hofgericht geprüft und anschließend dem Oberhofbeamten vorgelegt, der sie sammelt, nochmals prüft, geheim halten muss, um dann daraus nöthigenfalls aus eigener Erfahrung zu berichten ${ }^{166}$.

Es ist also ein ausgeklügeltes Kontrollsystem, das hier entwickelt wurde und das jeden Hofangehörigen im Grunde einer vollständigen Überwachung aussetzte, allerdings mit dem Vorteil, dass reine Gunstbeförderung oder aber aus Missgunst entstandene Herabsetzungen erschwert, wenngleich doch nicht unmöglich gemacht wurden.

\section{Der Burgfrieden und Burgfriedensbezirk in den württembergischen Hofordnungen}

Wie an den vorangegangenen Ausführungen gesehen, ist es keinesfalls so, dass der Burgfrieden und der Burgfriedensbezirk rein mittelalterliche oder frühneuzeitliche Thematiken oder Erscheinungen sind. Bis hinein ins 19. Jahrhundert

162 Württembergisches Staats- und Regierungs-Blatt (wie Anm.158) S.347f.

163 Ebd., S. 348.

164 Ebd., S.350f.

165 Ebd., S. 352.

166 Ebd. 
und wahrscheinlich bis zum Ende der höfischen Ära in Deutschland spielen sie eine wichtige Rolle im Strafrecht des Hofes.

Im Falle des Stuttgarter Burgfriedens und des dazugehörigen Burgfriedensbezirkes befinden wir uns in einer - aus Quellensicht - nahezu beispiellosen Situation, denn die württembergischen Hofordnungen gehen, wie geschildert, intensiv auf den Burgfriedensbezirk ein, beschreiben ihn detailliert und dies über Jahrhunderte hinweg, so dass auch die Entwicklung dieses Bezirks und die Veränderungen der Grenzen im Detail nachvollzogen werden können. Damit stehen die württembergischen Hofordnungen singulär da, denn keine andere bekannte Hofordnung enthält solche Beschreibungen, meist wird in diesen Quellen nur allgemein auf den Burgfrieden und das Vorhandensein eines Burgfriedensbezirkes verwiesen ${ }^{167}$.

Die erste württembergische Hofordnung, die den Stuttgarter Burgfriedensbezirk detailliert beschreibt, ist die Hofordnung Herzog Johann Friedrichs aus dem Jahr 1611. Der gleiche Text findet sich bis auf ganz geringe Abwandlungen auch in dessen Hofordnungen von 1614 und $1618^{168}$ :

Damit auch meniglich wißen möge, wie weitt der bezürkh des Burgfriedens sonderlich allhie zu Stuttgardten sich erstreckbe und sich der Unwißenheit niemandt zu entschuldigen habe, so wöllen wür solchen Burgfriedensbezürckh hiemit specifice benennt haben: namblich im ganzen Begriff unsers Schloß und Thiergartens, darunter auch der grab [en] von dem falckenhauß biß zum Thor gegen der Eßlinger Vorstatt hinauß, item in der Canzley wie nit weniger auch im ganzen begriff des neuen und alten Marstalls, Falkhen-, Vieh-, Jäger-, Zeug- und Bündthaus [1611 ergänzt durch: so weit selbiger (der Mauer nach) wegen unserm Landtschaftshauß übergehet, und von dannen ann unser Cammersecretarij Rhatgeber gartten und hause hinauswerts und dannen an die Probstey usw.] alß auch Baubof, Hofmeßigt und heuescheuer und insgemein von der Schloßbruckben an biß für daß inner Tentzlinger oder Canzleithor hinauß und von dannen in dem ganzen einschlu $\beta$ des Zeug- und Bündthauses biß an die Probstey und abermablen von dannen auff dem ganzen Kirchbofe hinüber bis zu der alten Keutzin [1611: deren von Kaltenthal Behausung, so unser Jagermeister bewohnt] hauß und also beschließlich biß wieder hinab zu obgemeltem Alt- und Neuem Marstall, wie wür dann deßwegen sonderlich Täfelin mablen und die an underschiedlichen orthen offentlich auffschlagen laßen.

Die Beschreibung des Burgfriedensbezirks ändert sich auch in den Hofordnungen Herzog Eberhard Ludwigs aus den Jahren 1660, 1685 und 1696 nicht entscheidend, und noch in der Hofordnung von 1711, die ebenfalls von Eberhard Ludwig

167 Unter den von mir untersuchten 122 deutschen Hofordnungen des 13. bis 19. Jahrhunderts finden sich genaue Beschreibungen des Burgfriedens und des Burgfriedensbezirkes nur in den württembergischen Hofordnungen. Es wäre allerdings von Interesse herauszufinden, ob es solche Beschreibungen auch in anderen Territorien zumindest zwischenzeitlich gab.

168 Alle drei Hofordnungen finden sich bei Kern (wie Anm.7), Bd.2, S. 143-160. 
stammt, sind die Grenzen des Burgfriedensbezirks anscheinend nahezu unverändert geblieben:

Damit nun männiglich wissen möge, wie weit der Bezirck des Burg-Fridens, sonderlich allhier zu Stuttgart, sich erstrecke, und sich der Unwissenheit niemand zu entschuldigen habe. So wollen Wir solch Burg-Fridens-Bezirck biemit specificè benannt haben: nemlich in ganzen Begriff Unsers Schlosses und thier-Gartens, darunter auch der Graben von dem Falcken-Haus, biß zu dem Thor, gegen der Eßlinger Vorstatte binaus; Item, in der Cantzley, wie nicht weniger auch in dem ganzen Begriff des neuen und alten Marstalls, Falcken-Vieb-Jäger-Zeug- und Bind-Haus, also auch Bau-Hof, Hofmetzig, und Heuschenern, und insgemein von der Schloß-Brucken an, das Cantzley-Thor hinaus, und von dannen auf den ganzen Kirch-Hof hinüber, biß zum Distlerischen Haus und also beschließlich biß wieder hinab zu obermeldtem alt-und neuen Marstall, wie Wir dann dessentwegen besondere Täfelein mablen, und die an unterschiedlichen Orten aufschlagen lassen; So vil die Determination des Bezircks des Burg-Fridens in hiesiger Unserer Residenz Ludwigsburg betrifft, solle solche nächstens erfolgen, und selbige mit Anschlagung besonderer Tafeln bemerckt werden ${ }^{169}$.

Mit dem Bau des neuen Schlosses, mit dem im Jahr 1746 begonnen wurde, ändern sich auch die Grenzen des Burgfriedensbezirks, wie an der Hofordnung Herzog Ludwig Eugens aus dem Jahr 1794 gesehen werden kann:

Damit Männiglich wissen möge, wie weit der Bezirk des Burgfrieden, sonderbeitlich zu Stuttgart sich erstreke, und sich der Unwissenheit dißfalls niemand $z u$ entschuldigen habe; so wollen Wir solche Burgfrieden-Bezirk hiemit benennt haben; nebmlich im ganzen Begriff des alt- und neuen Herzoglichen Schlosses, ebmaligen Militair-Academie-Gebäude, Opern- und Comödien-Hauß, die neue Anlagen zwischen beeden Herzoglichen Schloß, dem Waisenhauß und Münz, und überhaupt der ganze mit Bäumen ausgesetzte Plaz, der Herzogliche Marstall und Baubof, die Canzley, der sogenannte Prinzen-Bau und Fürstenhauß, deßgleichen der ganze Umfang des äussern Schloßplazes, welchen das alte Schloß, die Canzley, Prinzenbau, Herrschaftlicher Fruchtkasten, Stadt-Kirche, und das Caffeehauß einschliessen $^{170}$.

Herzog Friedrich II. übernahm diese Grenzen des Burgfriedensbezirkes für seine im Jahr 1798 erschienene Hofordnung ohne Veränderung ${ }^{171}$.

Selbst noch in der königlich württembergischen Hofordnung von 1807 findet sich der genaue Umfang des Burgfriedensbezirkes in Stuttgart detailliert beschrieben:

169 Moser (wie Anm. 4), Bd. 1, Anhang, S. 61.

170 Hofordnung Herzog Ludwig Eugen von Württemberg-Teck (wie Anm. 7) S.6f.

171 Hofordnung Herzog Friedrich II. von Württemberg-Teck (wie Anm.7) S.6. 
Der Umfang des Burgfriedens aber ist folgender:

Der ganze Innbegriff des alten und neuen Königl. Schlosses nebst des letzteren Nebengebäuden; der Bezirk des Königl. Palais auf dem Graben, das Opern- und Komödien-Haus, die neuen Anlagen zwischen beeden Königl. Schlössern, dem Waisenhaus und Münzgebäude und überhaupt der ganze mit Bäumen ausgesetzte Plaz. der Königl. Marstall und Baubof, die sämmtliche Kanzlei-Gebäude, der sogenannte Prinzenbau und Fürstenhaus, desgleichen der ganze Umfang des äussern Schloßplazes, welchen das alte Schloß, die Kanzlei, Prinzenbau, herrschaftlicher Fruchtkasten, Stadtkirche und das Caffe-Haus einschliessen. ${ }^{172}$

Und auch in der letzten württembergischen Hofordnung aus dem Jahr 1818 ist eine genaue Beschreibung dieses Burgfriedensbezirkes enthalten:

Der Bezirk des Burgfriedens wird begränzt:

a) in Stuttgart durch die Linie, welche vom Anfang der Planie längs der Königstraße und ibrer Fortsetzung außerhalb dem Thore, sodann längs der nach dem Königlichen Bad fübrenden Querstraße und ibrer Fortsetzung bis zum Kannstadter Thor sich hinziebt, die östliche Seite des Waisenhauses, das Ministerialgebäude des Innern, den alten Marstall, den Gasthof zum König von England, die Stadtkirche, den herrschaftlichen Fruchtkasten und den Prinzenbau berührt.

Die Straßen selbst, welche die Gränze bilden, sind nicht mehr im Burgfrieden begriffen, wohl aber die auf dieselben stoßenden zur Hofhaltung gehörigen Gebäude, als: der Prinzenbau, Hofstall und das Fürstenhaus;

b) außer Stuttgart sind besonders befriedet: alle diejenigen Gebäude, welche Seine Majestät der König und Ibre Majestät die Königin bewohnen, nebst den dazu gebörigen Gärten, Hofplätzen etc. jedoch nur für die Dauer des Aufenthalts Seiner Majestät des Königs und Ibrer Majestät der Königin ${ }^{173}$.

Die hier zitierten Auszüge aus württembergischen Hofordnungen der Jahre 1611 bis 1818 zeigen deutlich, dass Burgfriedensbezirke durchaus nicht nur den direkten Bereich des Schlosses oder der Burg umfassten, sondern durchaus auch Teile der angrenzenden Stadt mit beinhalten konnten, und dass sie überdies Veränderungen unterlagen und keinesfalls monolithisch waren. Insbesondere der Neubau eines Schlosses oder auch anderer Gebäude, die dem Bereich der Hofverwaltung zuzuordnen sind, war regelmäßig Anlass, den Burgfriedensbezirk zu erweitern bzw. zu modifizieren (Abb.5).

172 Reyscher (wie Anm.7), Bd.7, S. 88.

173 Hofordnung König Wilhelm von Württemberg 1818, in: Das Regierungs-Blatt für das Königreich Württemberg im Auszuge. Eine Sammlung sämmtlicher in den Regierungs-Blättern des Königreichs Württemberg vom Jahre 1806 an enthaltenen, noch ganz oder theilweise gültigen Gesetze, Verordnungen etc. mit erläuternden Anmerkungen und einem Haupt-Register 1840, S.71-77, hier S. 74. 


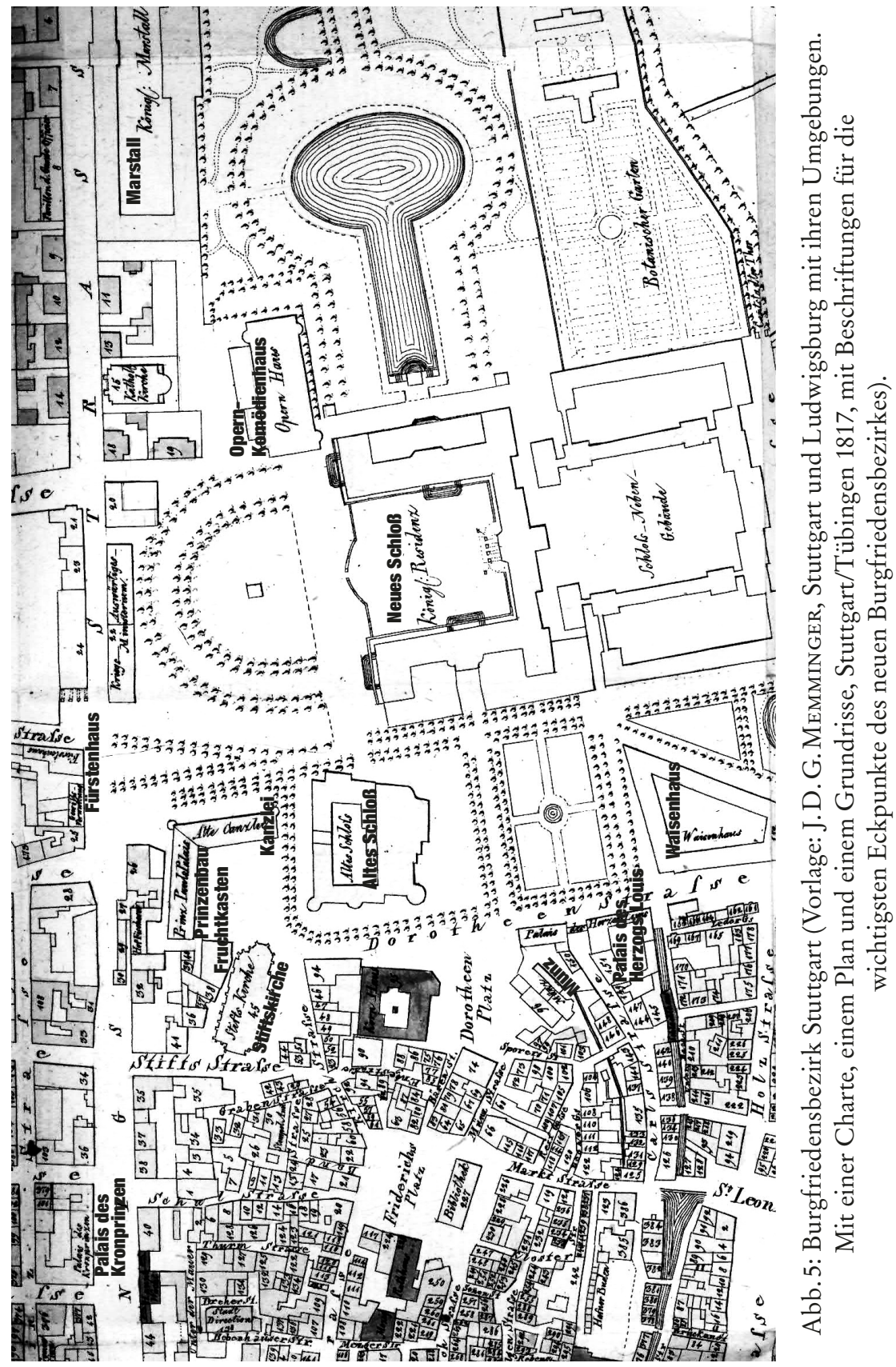




\section{Fazit}

Die vorgestellten Quellen machen deutlich, dass es sich sowohl beim Burgfrieden als auch beim Burgfriedensbezirk nicht um rein mittelalterliche Termini und Erscheinungen handelt, die spätestens mit dem Beginn der Neuzeit ad acta gelegt worden wären. Die Hofordnungen der württembergischen Herzöge und Könige, die bis zum Jahr 1818 die Grenzen eben jenes Stuttgarter Burgfriedensbezirkes und teilweise auch anderer Friedensbezirke detailliert beschreiben und auch stets aktualisieren, belegen deutlich, dass sowohl der Burgfrieden als auch der zu ihm gehörige Geltungsbereich bis zum Ende der deutschen Hofgeschichte von Bedeutung waren und eigenständige Rechtsbezirke darstellten, über deren Einhaltung und Wahrung die Fürsten akribisch wachten.

Die Untersuchung zu den frühneuzeitlichen und neuzeitlichen Burgfrieden und ihren Bezirken bleibt weiterhin ein Desiderat der Forschung; allerdings konnten die vorangegangenen Ausführungen zeigen, dass es durchaus zum Verständnis der Höfe und ihrer Geschichte beitragen kann, eben jene speziellen Rechtsverhältnisse und ihre Geltungsbereiche genauer zu untersuchen, auch und vor allem in Verbindung mit den gültigen Hofordnungen. 